\title{
Photoabsorption in formaldehyde: Intensities and assignments in the discrete and continuous spectral intervals
}

\author{
P. W. Langhoff \\ Department of Chemistry, Indiana University, Bloomington, Indiana 47401
}

A. E. Orel ${ }^{\mathrm{a})}$ and T. N. Rescigno

Theoretical Atomic and Molecular Physics Group, Lawrence Livermore Laboratory, University of California, Livermore, California 94550

\section{B. V. McKoy}

Arthur Amos Noyes Laboratory of Chemical Physics, California Institute of Technology, Pasadena, California 91125

(Received 13 June 1978)

\begin{abstract}
Theoretical investigations of total and partial-channel photoabsorption cross sections in molecular formaldehyde are reported employing the Stieltjes-Tchebycheff $(S-T)$ technique and separated-channel static-exchange (IVO) calculations. Vertical one-electron dipole spectra for the $2 b_{2}(n), 1 b_{1}(\pi), 5 a_{1}(\sigma)$, $1 b_{2}$, and $4 a_{1}$ canonical molecular orbitals are obtained using Hartree-Fock frozen-core functions and large basis sets of compact and diffuse normalizable Gaussians to describe the photoexcited and ejected electrons. The calculated discrete excitation spectra provide reliable zeroth-order approximations to both valence and Rydberg transitions, and, in particular, the $2 b_{2}(n) \rightarrow \mathrm{nsa}_{1}, \mathrm{npa}_{1}, \mathrm{npb}_{2}$, and nda ${ }_{2}$ IVO spectra are in excellent accord with recent experimental assignments and available intensity measurements. Convergent (S-T) photoionization cross sections in the static-exchange (IVO) approximation are obtained for the 15 individual partial channels associated with ionization of the five occupied molecular orbitals considered. Resonance features in many of the individual-channel photoionization cross sections are attributed to contributions from valencelike $a_{1} \sigma^{*}(\mathrm{CO}), a_{1} \sigma^{*}(\mathrm{CH})$, and $b_{2} \sigma^{*}(\mathrm{CH}) / \pi_{y} *(\mathrm{CO})$ molecular orbitals that appear in the photoionization continua, rather than in the corresponding one-electron discrete spectral intervals. The vertical electronic cross sections for ${ }^{1} A_{1} \rightarrow^{1} B_{1},{ }^{1} B_{2}$, and ${ }^{1} A_{1}$ excitations are in generally good accord with previously reported $\mathrm{CI}(\mathrm{S}-\mathrm{T})$ predictions of continuum orbital assignments and intensities, although some discrepancies due to basis-set differences are present in the ${ }^{1} B_{1}$ and ${ }^{1} B_{2}$ components, and larger discrepancies apparently due to channel coupling are present in the ${ }^{1} A_{1} \rightarrow{ }^{1} A_{1}$ cross section. Partialchannel vertical electronic cross sections for the production of the five lowest parent-ion electronic states are found to be in general agreement with the results of very recent synchrotron-radiation photoelectron branching-ratio measurements in the 20 to $30 \mathrm{eV}$ excitation energy interval. Most important in this connection is the tentative verification of the predicted orderings in intensities of the partial-channel cross sections, providing support for the presence of a strong $k a_{1} \sigma^{*}(\mathrm{CO})$ resonance in the $\left(5 a_{1}^{-1}\right)^{2} A_{1}$ channel. Finally, the total vertical electronic cross sections for absorption and ionization are in general accord with photoabsorption measurements, photoionization-mass-spectrometric studies, and the previously reported CI (S-T) calculations. Although further refined calculations including vibrational degrees of freedom and autoionization line shapes are required for a more precise quantitative comparison between theory and experiment, the present study should provide a reliable zeroth-order account of discrete and continuum electronic dipole excitations in molecular formaldehyde.
\end{abstract}

\section{INTRODUCTION}

Photoabsorption and related phenomena in molecular formaldehyde have been the subject of continuing experimental ${ }^{1-11}$ and theoretical ${ }^{12-28}$ study. In spite of this attention, certain aspects of the spectrum apparently require further investigation. The $n=3$ and other members of the higher (autoionizing) Rydberg series, for example, are not observed, ${ }^{4,5}$ the missing $\pi \rightarrow \pi^{*}$ transition is a continuing puzzle, ${ }^{7,11}$ and few theoretical attempts have been made to clarify intensity distributions in the photoionization continuum. ${ }^{28}$ In view of its historical significance and prototypical importance, ${ }^{7}$ a detailed theoretical study of both discrete and continuum electronic excitations in molecular formaldehyde at a reliable computational level of approximation would seem to be desirable.

\footnotetext{
a)Present address: Department of Chemistry, University of California, Berkeley, CA 94720.
}

In the present paper, theoretical investigations of total and partial-channel photoabsorption/ionization cross sections in molecular formaldehyde are reported employing the previously devised Stieltjes-Tchebycheff $(S-T)$ technique in the separated-channel staticexchange (IVO) approximation. ${ }^{29-31}$ Pseudospectra of discrete transition frequencies and oscillator strengths appropriate for one-electron vertical dipole excitation of the $2 b_{2}(n), 1 b_{1}(\pi), 5 a_{1}(\sigma), 1 b_{2}$, and $4 a_{1}$ canonical molecular orbitals are constructed employing frozen HartreeFock core functions and large basis sets of compact and diffuse normalizable Gaussians to describe the photoexcited and ejected electrons. These pseudospectra provide approximations to both discrete and continuum excitations in formaldehyde when used in conjunction with the $S-T$ technique. ${ }^{32-39}$ The calculated discrete IVO excitations furnish reliable zeroth-order approximations to both valence and Rydberg transitions. In particular, the $2 b_{2}(n) \rightarrow n s a_{1}, n p a_{1}, n p b_{2}$, and $n d a_{2}$ series are found to be in good agreement with available position and in- 
tensity measurements, and with the results of previous theoretical studies. Although the predicted higher Rydberg series are in accord with the tentatively assigned spectral positions, ${ }^{5}$ autoionizing line shape calculations are required to clarify the observed intensities. Convergent vertical electronic IVO $(S-T)$ photoionization cross sections are obtained for the 15 individual partial channels associated with ionization of the $2 b_{2}(n), 1 b_{1}(\pi)$, $5 a_{1}(\sigma), 1 b_{2}$, and $4 a_{1}$ molecular orbitals. Many of the partial-channel cross sections exhibit resonance features that can be attributed to the presence of valencelike $a_{1} \sigma^{*}(\mathrm{CO}), a_{1} \sigma^{*}(\mathrm{CH})$, and $b_{2} \sigma^{*}\left(\mathrm{CH} / \pi_{y}^{*}(\mathrm{CO})\right.$ molecular orbitals in the photoionization continuum. The component profiles are employed in constructing vertical electronic photoabsorption cross sections for ${ }^{1} A_{1}-{ }^{1} B_{1},{ }^{1} B_{2}$, and ${ }^{1} A_{1}$ symmetries, partial-channel cross sections for the production of the five lowest parent ion electronic states, and the total cross sections for absorption and ionization. Detailed comparisons are made of these profiles with the results of previous CI (S-T) calculations, ${ }^{28}$ with recent synchrotron-radiation branchingratio measurements, and with total absorption and photoionization-mass-spectrometric studies. 4,8 The IVO and $\mathrm{CI}$ results for the three polarization cross sections are in general accord, although there are some small discrepancies in the respective ${ }^{1} A_{1} \rightarrow{ }^{1} B_{1}$ and ${ }^{1} B_{2}$ components due to basis set differences, and larger discrepancies are present in the ${ }^{1} A_{1}-{ }^{1} A_{1}$ component, apparently due to the effects of channel coupling. The calculated IVO partial-channel cross sections are in good agreement with recent synchrotron-radiation branching-ratio measurements in the 20 to $30 \mathrm{eV}$ excitation interval, and the photoelectron spectra provide tentative verification of a predicted strong $5 a_{1}-k a_{1} \sigma^{*}(\mathrm{CO})$ resonance feature in the $\left(5 a_{1}^{-1}\right)^{2} A_{1}$ photoionization channel. The presence of such resonance features in the photoionization cross sections, and their clarification in terms of conventional $\sigma^{*}$ and $\pi^{*}$ valencelike orbitals in the photoionization continua, is perhaps one of the more noteworthy aspects of the present study. Finally, the cross sections for absorption and ionization obtained from the IVO and CI calculations are in mutual accord, and in general agreement with the results of total absorption and photoionization-mass-spectrometric studies.

A brief description of the $\mathrm{S}-\mathrm{T}$ technique is provided for completeness in Sec. II, although the reader is encouraged to consult earlier more detailed reports for complete discussion of the approach. ${ }^{32-39}$ The separated-channel static-exchange (IVO) approximation is described in Sec. III, and the discrete basis sets employed and calculations made in the case of formaldehyde are described in Sec. IV. The resulting photoabsorption cross sections are described and compared with previous theoretical studies and experimental results in Sec. V, and concluding remarks are made in Sec. VI.

\section{STIELTJES-TCHEBYCHEFF TECHNIQUE}

The Stieltjes-Tchebycheff (S-T) technique provides a general method for constructing accurate approximations to the discrete and continuum vertical electronic portions of molecular photoabsorption cross sections from discrete pseudospectra of transition frequencies and oscillator strengths ${ }^{40}$

$$
\left(\tilde{\epsilon}_{i}, \tilde{f}_{i}\right), \quad i=1,2, \cdots, N,
$$

calculated employing only $L^{2}$ functions of good symmetry type and some appropriate computational approximation. In this way, difficulties in constructing molecular continuum functions of good symmetry are avoided, and the general technology of computational quantum chemistry is brought to bear on molecular photoionization problems. The frequencies and strengths of Eq. (1) can refer to the total vertical electronic photoabsorption cross section of a molecule, to the cross section for a particular polarization direction in the molecular frame, or to the partial-channel cross section associated with the production of a specific final ionic state. Moreover, the level of approximation employed in calculating the pseudospectrum of Eq. (1) can range from simple one-electron local-potential treatments to detailed configuration-interaction studies. ${ }^{28-39}$

Bin-smoothing approaches, corresponding to finiteenergy-interval averaging of the primitive pseudospectrum [Eq. (1)], can also be employed in constructing smooth profiles, although these generally give very poor vertical electronic cross sections when applied to the somewhat irregular pseudospectra [Eq. (1)] obtained employing nonlocal potentials or configuration-interaction calculations. In the $\mathrm{S}-\mathrm{T}$ technique, the spectral moments $^{40}$

$$
\tilde{S}_{N}(-k)=\sum_{i=1}^{N} \tilde{f}_{i} \tilde{\epsilon}_{i}^{-k}, \quad k=0,1, \ldots, 2 n-1,
$$

$(n \ll N)$ are constructed, and these are employed in calculations of moment-theory spectra

$$
\left[\epsilon_{i}(n), f_{i}(n)\right], \quad i=1,2, \ldots, n,
$$

which satisfy

$$
\tilde{S}_{N}(-k)=\sum_{i=1}^{n} f_{i}(n) \epsilon_{i}(n)^{-k}, \quad k=0,1, \ldots, 2 n-1 .
$$

When sufficiently large $L^{2}$ basis sets are employed, the $\tilde{S}_{N}(-k)$ furnish accurate approximations to the correct invariant spectral moments $S(-k)$ of the target system. ${ }^{34,36}$ In this case, the spectrum of Eq. (3) can be employed in determining the correct molecular photoexcitation and ionization cross section. Specifically, bounding approximations are constructed to the cumulative oscillator-strength distribution, and these are differentiated in the S-T sense with respect to the energy variable. ${ }^{36,37}$ The oscillator-strength density so obtained exhibits resonancelike structures at the appropriate discrete transition frequencies, and is smooth in the photoionization region. ${ }^{32-39}$ The strengths of the discrete transitions are obtained by constructing the so-called Stieltjes histogram, which provides a generalization of the familiar Coulomb extrapolation at the ionization limit. ${ }^{33,34}$

Other appropriately defined integrals or generalized moments can also be employed in conjunction with the S-T technique as alternatives to the negative-integer power moments of Eq. (2). ${ }^{41,42}$ Moreover, in computational applications, the recurrence coefficients 


$$
\left(\alpha_{k}, \beta_{k-1}\right), \quad k=0, \ldots, n-1,
$$

for the polynomials orthogonal with respect to the oscillator-strength density are calculated directly from the primitive spectrum of Eq. (1) employing a stable computational algorithm, ${ }^{35,36}$ avoiding explicit solution of Eq. (4). The moment-theory spectrum [Eq. (3)] is then obtained from the poles and residues of appropriately defined orthogonal-polynomial rational fractions. ${ }^{37}$

The recurrence coefficients of Eq. (5) provide criteria useful for estimating the convergence of quantummechanical calculations of the primitive pseudospectrum $[E q$. (1)]. Moreover, the calculated coefficients can be supplemented with additional $\alpha_{k} \beta_{k}$ values chosen to extrapolate smoothly in $k$ to the asymptotic expressions

$$
\begin{aligned}
& \alpha_{\infty}=1 /\left(2 \epsilon_{t}\right), \\
& \beta_{\infty}=1 /\left(4 \epsilon_{t}\right)^{2},
\end{aligned}
$$

where $\epsilon_{t}$ is the threshold for photoionization. ${ }^{36}$ The specific choice of extrapolation function employed depends to a certain extent on the overall shape of the photoionization spectrum treated, although experience indicates the results are highly insensitive to choice when sufficient numbers of calculated coefficients are employed. ${ }^{36}$ In this way, a complete set of recurrence coefficients is obtained, and the $\mathrm{S}-\mathrm{T}$ technique can provide completely convergent oscillator-strength distributions and densities.

\section{SEPARATED-CHANNEL STATIC-EXCHANGE APPROXIMATION}

In the Born-Oppenheimer approximation, the vertical electronic photoabsorption cross section of a molecule is calculated in a body-fixed coordinate system and written as a sum of partial cross sections for the energetically open channels

$$
\sigma(\omega)=\sum_{\Gamma} \sigma_{\Gamma}(\omega)
$$

where

$$
\sigma_{\Gamma}(\omega)=\left(2 \pi^{2} / c\right) g_{\Gamma}(\omega),
$$

and

$$
g_{\Gamma}(\omega)=(2 / 3) \omega\left|\left\langle\Psi_{\Gamma}^{-}|\mu| \Psi_{0}\right\rangle\right|^{2}
$$

is the oscillator-strength density for the channel $\Gamma{ }^{40}$ In Eq. (9), $\Psi_{\Gamma}^{-}$represents any of the unity-normalized bound electronic states of the molecule $\left(\omega=\epsilon_{i}-\epsilon_{0}\right)$, or refers to a molecular continuum function $\left(\omega=\epsilon-\epsilon_{0}\right)$ satisfying incoming boundary conditions appropriate for an electron incident upon the ionic state associated with the channel $\Gamma$. The functions $\Psi_{\Gamma}^{-}$generally include appropriate combinations of various valence configurations and Rydberg states in the discrete region of the spectrum. In the continuum, they contain scattered-wave components that give the inelastic amplitudes for scattering in all other open channels, and include the various autoionizing states associated with Rydberg levels above the lowest ionization threshold. Of course, the electronic functions $\Psi_{\Gamma}^{-}$defined in a body-fixed coordinate system generally do not correspond to a single partial wave having definite electronic angular momentum.
Consequently, the $\Psi_{\Gamma}^{-}$satisfy differential equations associated with energetically coupled channels each of which also involves electronic angular-momentum coupling in the molecule-fixed frame.

Previous theoretical studies of photoexcitation and ionization in molecular nitrogen and carbon monoxide suggest that the problem of energetic channel coupling can be overcome simply by employing a one-electron approximation that identifies the important physically distinct electronic excitation mechanisms expected to dominate the photoabsorption cross section of the target system. ${ }^{29-31}$ In this way, one-electron excitations are identified for which nonlocal molecular static-exchange potentials can be constructed employing Hartree-Fock wavefunctions for the appropriate core states. This approximation neglects both energetic channel coupling and the effects of core relaxation upon photoexcitation. Some allowance is made in the development, however, for the use of a frozen core by employing experimental, rather than Koopmans, ionization potentials in each case. The angular-momentum coupling problem within each electronic channel is solved completely by employ ing functions of good molecular symmetry type and the $S-T$ technique. Channel coupling effects can also be investigated in the context of $S-T$ calculations, and are treated in a separate study.

The electronic ground state of formaldehyde is described in the Hartree-Fock approximation in terms of canonical orbitals in the form

$$
\left(1 a_{1}^{2} 2 a_{1}^{2} 3 a_{1}^{2} 4 a_{1}^{2} 1 b_{2}^{2} 5 a_{1}^{2} 1 b_{1}^{2} 2 b_{2}^{2}\right) X^{1} A_{1} .
$$

Following the separated-channel approximation, there are $\mathbf{1 5}$ distinct one-electron dipole series corresponding to individual excitations of the five highest-lying molecular orbitals. These series are designated

$$
\begin{array}{lll}
\left(2 b_{2}^{-1} k a_{2}\right)^{1} B_{1}, & \left(2 b_{2}^{-1} k a_{1}\right)^{1} B_{2}, & \left(2 b_{2}^{-1} k b_{2}\right)^{1} A_{1}, \\
\left(1 b_{1}^{-1} k a_{1}\right)^{1} B_{1}, & \left(1 b_{1}^{-1} k a_{2}\right)^{1} B_{2}, & \left(1 b_{1}^{-1} k b_{1}\right)^{1} A_{1}, \\
\left(5 a_{1}^{-1} k b_{1}\right)^{1} B_{1}, & \left(5 a_{1}^{-1} k b_{2}\right)^{1} B_{2}, & \left(5 a_{1}^{-1} k a_{1}\right)^{1} A_{1}, \\
\left(1 b_{2}^{-1} k a_{2}\right)^{1} B_{1}, & \left(1 b_{2}^{-1} k a_{1}\right)^{1} B_{2}, & \left(1 b_{2}^{-1} k b_{2}\right)^{1} A_{1}, \\
\left(4 a_{1}^{-1} k b_{1}\right)^{1} B_{1}, & \left(4 a_{1}^{-1} k b_{2}\right)^{1} B_{2}, & \left(4 a_{1}^{-1} k a_{1}\right)^{1} A_{1} .
\end{array}
$$

In each of these cases, one-electron problems of the form

$$
\left(h_{\Gamma}-\epsilon\right) \phi_{\epsilon}^{\Gamma}=0
$$

are obtained, where

$$
h_{\Gamma}=T+V+\sum_{i}\left(2 J_{i}-K_{i}\right)+J_{\Gamma}+K_{\Gamma}
$$

is the appropriate static-exchange Hamiltonian. ${ }^{43}$ In Eq. (13), $T$ and $V$ refer to the kinetic-energy and nuclear-framework potential-energy operators, respectively, the sum $i$ is over the Coulomb $J_{i}$ and exchange $K_{i}$ operators for the doubly occupied core orbitals, and $\phi_{\epsilon}^{\Gamma}$ refers to the singly excited orbital under consideration. Since the one-electron Hamiltonian of Eqs. (12) and (13) contains a $V^{(N-1)}$ potential, ${ }^{44,45}$ the bound states of Eq. (12) correspond to so-called improved virtual orbitals (IVO). ${ }^{43}$ These can be expected to provide useful first approximations to valence and Rydberg excita- 
tions in formaldehyde when based on canonical HF orbitals, and the associated continuum states are expected to provide correspondingly reliable approximations to the one-electron photoionization spectra. ${ }^{46}$

Since the Hamiltonian of Eq. (13) is Hermitian, the solutions of Eq. (12) form an orthogonal set. However, the lowest energy bound states obtained from Eq. (12), corresponding to the occupied orbitals of appropriate symmetry, generally differ from those of Eq. (10), since the latter are obtained employing a Hartree-Fock Hamiltonian different from that of Eq. (13). In order to employ the conventional ground-state occupied canonical Hartree-Fock orbitals in the calculations, and to deal only with orthonormal orbitals, it is convenient to explicitly orthogonalize the solutions of Eq. (12) to the occupied canonical orbitals in each case. Procedures for this are described in the following section.

\section{DISCRETE BASIS SET CALCULATIONS}

The potentials appearing in Eqs. (12) and (13) are noncentral and nonlocal, necessitating the introduction of special computational procedures in their solution. Although such equations can be solved in a variety of ways for simple systems, such as molecular hydrogen, convergence difficulties can arise for larger more anisotropic molecules. In the present studies, Eqs. (12) and (13) are solved employing the Roothaan-Hartree-Fock procedure, and the $\mathrm{S}-\mathrm{T}$ technique is applied to the resulting discrete pseudospectra of good molecular symmetry. In this way, photoexcitation and ionization cross sections are obtained in formaldehyde in the separatedchannel static-exchange (IVO) approach without further computational approximation, yet the explicit construction of continuum functions of correct molecular symmetry is avoided.

A Hartree-Fock function [Eq. (10)] of good quality is constructed near the equilibrium internuclear geometry in formaldehyde employing $(4 s) /[2 s]$ hydrogen, $(10 s, 5 p$, $1 d) /[3 s, 2 p, 1 d]$ carbon, and $(10 s, 5 p, 1 d) /[3 s, 2 p, 1 d]$ oxygen basis sets of contracted Gaussian functions. ${ }^{20}$ The spatial characteristics of the occupied canonical HF orbitals have been described in previous publications. ${ }^{47}$ Further description of these orbitals, and of the associated virtual valencelike orbitals obtained, is given at appropriate points below. The occupied canonical orbitals are used to construct the individual channel Hamiltonians of Eq. (13), and discrete-basis-set expansions are employed to obtain variational solutions of Eq. (12) satisfying

$$
\begin{aligned}
& \left\langle\phi_{i}^{\Gamma}\left|h_{\Gamma}\right| \phi_{j}^{\Gamma}\right\rangle=\epsilon_{i}^{\Gamma} \delta_{i j}, \\
& \left\langle\phi_{i}^{\Gamma} \mid \phi_{j}^{\Gamma}\right\rangle=\delta_{i j}, \quad i, j=1,2, \cdots, N .
\end{aligned}
$$

Associated pseudospectra of transition frequencies and oscillator strengths are obtained from Eqs. (14) and the expressions

$$
\begin{aligned}
& \tilde{\epsilon}_{i}^{\Gamma}=\epsilon_{\Gamma}+\epsilon_{i}^{\Gamma}, \\
& \tilde{f}_{i}^{\Gamma}=(2 / 3) \tilde{\epsilon}_{i}^{\Gamma}\left|\left\langle\phi_{i}^{\Gamma}|\mu| \phi_{\Gamma}\right\rangle\right|^{2},
\end{aligned}
$$

where the experimental ionization potential is used for $\epsilon_{\Gamma}, \phi_{\Gamma}$ is the occupied orbital of the active electron in the channel $\Gamma$, and $\mu$ is the dipole moment operator. The spectra of Eqs. (15) are then employed in the S-T technique in construction of the photoabsorption/ionization cross sections. ${ }^{32-39}$

Large basis sets of compact and diffuse Gaussian functions are employed in the construction of solutions in the form of Eqs. (14). The virtual orbitals obtained from the ground-state Hartree-Fock calculation are supplemented with the additional compact and diffuse functions indicated in Table I. This augmented basis set is explicitly orthogonalized to the occupied Fock canonical orbitals prior to constructing solutions in the form of Eqs. (14), providing IVO spectra comprised of $45 a_{1}$ orbitals, $19 a_{2}$ orbitals, $30 b_{1}$ orbitals, and $32 b_{2}$ orbitals. The calculations should provide good approximations to the frequencies and strengths of the first few members of the various discrete excitation series. Moreover, the pseudospectra are sufficiently dense in the continuum excitation regions to provide convergent IVO $\mathrm{S}-\mathrm{T}$ photoionization cross sections. In view of the relatively large number of compact and diffuse basis functions employed in the present calculation, it is highly unlikely that the use of additional basis functions will result in significant changes in the calculated formaldehyde photoabsorption cross sections in the IVO $\mathrm{S}-\mathrm{T}$ approximation. Specific potential effects of basis function deficiency, however, are discussed at appropriate points in the text.

\section{ELECTRONIC EXCITATION SPECTRA IN FORMALDEHYDE}

In this section, descriptions are provided of (A) the discrete and continuum IVO S-T individual-orbital vertical electronic excitation spectra in $\mathrm{H}_{2} \mathrm{CO}$, (B) the resonances appearing in channels having $k a_{1}$ and $k b_{2}$ finalstate orbital symmetry, (C) the total vertical electronic cross sections for the three independent electric-field polarization directions, (D) the partial-channel vertical electronic cross sections for the production of the five lowest-lying electronic parent-ion states, and $(\mathrm{E})$ the total vertical electronic photoabsorption and photoionization cross sections. These results are compared with relevant experimental determinations and previous theoretical studies at appropriate points in the text.

Prior to describing the results of the present IVO S-T calculations, it is perhaps helpful to recall that the $\mathrm{H}_{2} \mathrm{CO}$ canonical $\mathrm{HF}$ orbitals can be written approximately in the forms $1 a_{1}=a_{1} 1 s(\mathrm{O}), 2 a_{1}=a_{1} 1 s(\mathrm{C}), 3 a_{1}=a_{1} 2 s(\mathrm{O})$, $4 a_{1}=a_{1} \sigma(\mathrm{CH}), 1 b_{2}=b_{2} \sigma(\mathrm{CH}), 5 a_{1}=a_{1} \sigma(\mathrm{CO}), 1 b_{1}=b_{1} \pi(\mathrm{CO})$, and $2 b_{2}=b_{2} n(\mathrm{O})$, with more complete spatial characterizations given in previous publications. ${ }^{47}$ In addition to these occupied canonical Fock orbitals, calculations in a minimal or valence basis set will result in virtual or unoccupied Fock orbitals that can be characterized approximately in the forms $2 b_{1}=b_{1} \pi^{*}(\mathrm{CO}), 6 a_{1}=a_{1} \sigma^{*}(\mathrm{CO})$, $7 a_{1}=a_{1} \sigma(\mathrm{CH})$, and $3 b_{2}=b_{2} \sigma^{*}(\mathrm{CH}) / \pi_{y}^{*}(\mathrm{CO}){ }^{24}$ On the basis of the general natures of these orbitals, it is possible in the following to distinguish between intravalence transitions among relatively compact occupied and virtual orbitals on the one hand, and transitions into more dif- 
TABLE I. Gaussian basis functions employed in $\mathrm{H}_{2} \mathrm{CO}$ IVO calculations. ${ }^{2}$

\begin{tabular}{llll}
\hline \hline Location $^{\mathrm{b}}$ & Type & Number & Exponent range $^{\mathrm{c}}$ \\
\hline & & \multicolumn{2}{c}{$a_{1}$ symmetry } \\
$\mathrm{H}_{1}$ and $\mathrm{H}_{2}$ & $p_{y}, p_{z}$ & 1,1 & $0.8,0.8$ \\
$\mathrm{C}$ & $s, p_{z}, d_{z z}$ & $4,4,2$ & $0.1-2.0,0.1-2.0,0.2-0.4$ \\
$\mathrm{O}$ & $s, p_{z}, d_{z z}$ & $4,4,2$ & $0.1-2.0,0.1-2.0,0.2-0.4$ \\
c. m. & $s, p_{z}, d_{z z}$ & $6,6,2$ & $0.00625-0.2,0.00625-0.2,0.2-0.4$
\end{tabular}

$\begin{array}{lll}\mathrm{H}_{1} \text { and } \mathrm{H}_{2} & p_{x} & \\ \mathrm{C} & d_{x y} & \\ \mathrm{O} & d_{x y} & \\ \mathrm{c} . \mathrm{m} . & d_{x y} & 10\end{array}$

$a_{2}$ symmetry

$\begin{array}{llr}\mathrm{H}_{1} \text { and } \mathrm{H}_{2} & p_{x} & 1 \\ \mathrm{C} & p_{x}, d_{x z} & 4,2 \\ \text { O } & p_{x}, d_{x z} & 4,2 \\ \text { c. m. } & p_{x}, d_{x z} & 10,2\end{array}$
0.8
$0.1-0.75$
$0.1-0.85$
$0.00039-0.2$

$b_{1}$ symmetry
0.8
$0.1-2.0,0.2-0.4$
$0.1-2.0,0.2-0.4$
$0.00039-0.2,0.2-0.4$

$b_{2}$ symmetry

$\begin{array}{llrl}\mathrm{H}_{1} \text { and } \mathrm{H}_{2} & p_{y}, p_{z} & 1,1 & 0.8,0.8 \\ \mathrm{C} & p_{y}, d_{y z} & 4,2 & 0.1-2.0,0.2-0.4 \\ \mathrm{O} & p_{y}, d_{y z} & 4,2 & 0.1-2.0,0.2-0.4 \\ \text { c. m. } & p_{y}, d_{y z} & 10,2 & 0.00039-0.2,0.2-0.4\end{array}$

Supplemental functions employed, in addition to the virtual Fock basis indicated in Sec. IV, in the solution of Eqs. (12).

"The molecular geometry employed is that cited by D. L. Yeager and V. McKoy, J. Chem. Phys. 60, 2714 (1974). See also T. H. Dunning, Jr. and N. W. Winter, J. Chem. Phys. 55, 3360 (1971).

${ }^{c} A$ geometric series variation in the exponent range is employed when appropriate.

fuse Rydberg-like orbitals, resulting from the supplemental basis of Table I, on the other hand.

\section{A. Individual orbital-excitation spectra}

The discrete IVO excitation spectra obtained from solution of Eqs. (12) using the basis sets described in Sec. IV and Table I are summarized in Fig. 1, and numerical values of the individual transition frequencies and oscillator strengths are given in Tables II to IV. Experimentally determined vertical ionization potentials are used in Eqs. (15) for the $2 b_{2}, 1 b_{1}$, and $5 a_{1}$ orbitals, $^{2}$ whereas Koopmans values are used for the $1 b_{2}$ and $4 a_{1}$ orbitals because of apparent uncertainties in the experimental assignments.

Figure 1 provides an overall semiquantitative picture of the vertical electronic IVO dipole excitations in $\mathrm{H}_{2} \mathrm{CO}$. Detailed discussions of the individual spectra are provided in the following subsections. It is appropriate to make some general observations here, however, concerning these results. In particular, two separate ( $s$ and $p$ ) Rydberg series are evident in all the $n a_{1}$ finalstate symmetries in the figure, although there are no

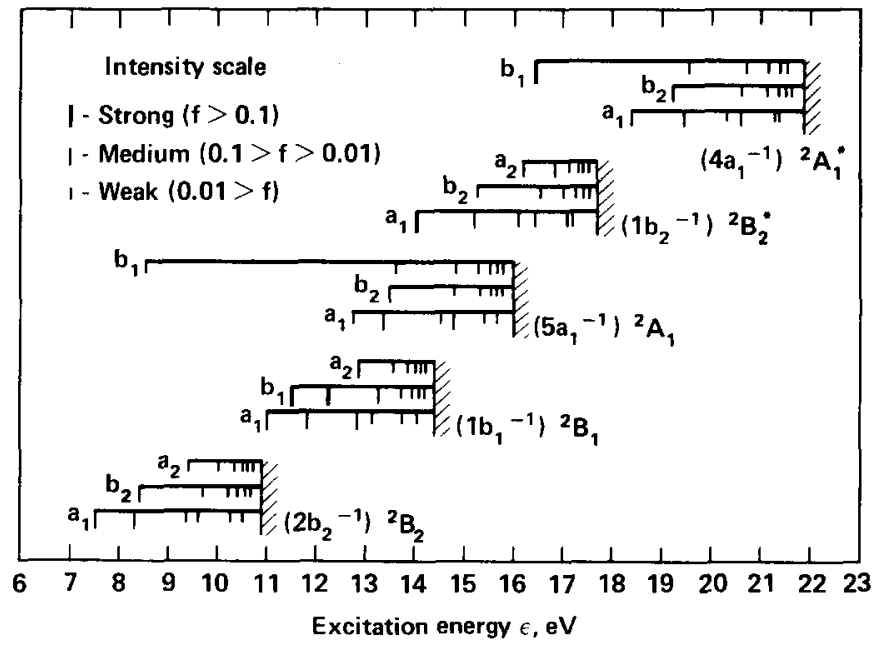

FIG. 1. Improved-virtual-orbital excitation spectra in $\mathrm{H}_{2} \mathrm{CO}$ obtained from solution of Eqs. (12) employing the basis sets described in Sec. IV and Table I. The lengths of the transition lines give semiquantitative indications of the $f$-number values in each case: strong, $f>0.1$; medium, $0.1>f>0.01$; weak, $0.01>f$. Numerical values of the transition frequencies and oscillator strengths are given in Tables II-IV. 
TABLE II. $2 b_{2}(n)$ excitation spectra in $\mathbf{H}_{2} \mathrm{CO}$ (I. P. $\left.=10.9 \mathrm{eV}\right)$.

\begin{tabular}{|c|c|c|c|c|c|}
\hline \multicolumn{2}{|c|}{ Present results ${ }^{a}$} & \multicolumn{2}{|c|}{ Experimental values $^{b, c}$} & \multicolumn{2}{|c|}{ Previous calculations $^{d}$} \\
\hline & $\begin{array}{l}\text { Energy }(\mathrm{eV}) / \\
f \text { number }\end{array}$ & & $\begin{array}{l}\text { Energy (eV)/ } \\
\text { intensity }\end{array}$ & & $\begin{array}{l}\text { Energy }(\mathrm{eV}) / \\
f \text { number }\end{array}$ \\
\hline${ }^{1} B_{2}\left(6 a_{1}\right)$ & $7.49 / 0.0149$ & ${ }^{1} B_{2}\left(3 s a_{1}\right)$ & $7.09 / f \sim 0.03$ & ${ }^{1} B_{2}\left(3 s a_{1}\right)$ & $7.16,7.56 / 0.007, \cdots$ \\
\hline$\left(7 a_{1}\right)$ & $8.29 / 0.0207$ & $\left(3 p a_{1}\right)$ & $8.13 / f \sim 0.03$ & $\left(3 p a_{1}\right)$ & $8.08,8.40 / 0.014, \cdots$ \\
\hline$\left(8 a_{1}\right)$ & $9.36 / 0.00557$ & $\left(4 s a_{1}\right)$ & $9.26 /$ weak & & $\cdots$ \\
\hline$\left(9 a_{1}\right)$ & $9.61 / 0.00769$ & $\left(4 p a_{1}\right)$ & $9.63 / \cdots$ & & $\cdots$ \\
\hline$\left(10 a_{1}\right)$ & $10.24 / 0.00220$ & $\left(5 s a_{1}\right)$ & $9.98 / \cdots$ & & $\cdots$ \\
\hline$\left(11 a_{1}\right)$ & $10.48 / \cdots$ & $\left(5 p a_{1}\right)$ & $10.13 / \cdots$ & & $\cdots$ \\
\hline${ }^{1} A_{1}\left(3 b_{2}\right)$ & $8.40 / 0.0220$ & ${ }^{\mathrm{t}} A_{1}\left(3 p b_{2}\right)$ & $7.97 / f \sim 0.02$ & ${ }^{1} A_{1}\left(3 p b_{2}\right)$ & $8.09,8.42 / 0.031, \cdots$ \\
\hline$\left(4 b_{2}\right)$ & $9.68 / 0.00797$ & $\left(4 p b_{2}\right)$ & $9.58 /$ weak & $\left(3 d b_{2}\right)$ & $9.23,9.50 / 0.0005, \cdots$ \\
\hline$\left(5 b_{2}\right)$ & $10.17 / 0.00376$ & $\left(5 p b_{2}\right)$ & $10.13 / \cdots$ & & $\cdots$ \\
\hline$\left(6 b_{2}\right)$ & $10.42 / 0.00207$ & $(6 p)$ & $10.39 / \cdots$ & & $\cdots$ \\
\hline$\left(7 b_{2}\right)$ & $10.55 / 0.00131$ & $(7 p)$ & $10.53 / \cdots$ & & $\cdots$ \\
\hline$\left(8 b_{2}\right)$ & $10.66 / \cdots$ & $(8 p)$ & $10.62 / \cdots$ & & $\cdots$ \\
\hline${ }^{1} B_{1}\left(1 a_{2}\right)$ & $9.36 / 0.000954$ & ${ }^{1} B_{1}(3 d)$ & $8.88 /$ weak & ${ }^{1} B_{1}\left(3 d a_{2}\right)$ & $9.21,9.47 / 0.0002, \cdots$ \\
\hline$\left(2 a_{2}\right)$ & $10.02 / 0.000506$ & $(4 d)$ & $9.84 / \cdots$ & & $\cdots$ \\
\hline$\left(3 a_{2}\right)$ & $10.33 / 0.000277$ & $(5 d)$ & $10.25 / \cdots$ & & $\cdots$ \\
\hline$\left(4 a_{2}\right)$ & $10.50 / 0.000162$ & $(6 d)$ & $10.46 / \cdots$ & & $\cdots$ \\
\hline$\left(5 a_{2}\right)$ & $10.60 / 0.000063$ & $(7 d)$ & $10.58 / \cdots$ & & $\cdots$ \\
\hline$\left(6 a_{2}\right)$ & $10.72 / \cdots$ & & & & \\
\hline
\end{tabular}

${ }^{2}$ Formaldehyde IVO spectra were obtained from Eqs. (12) and the basis sets described in Sec. IV and Table I. Principal quantum numbers refer to IVO energy ordering.

Experimental transition frequencies, corresponding to $0-0$ vibrational bands, $f$ numbers, and assignments taken from D. C. Moule and A. D. Walsh, Chem. Rev. 75, 67 (1975); C. R. Lessard and D. C. Moule, J. Chem. Phys. 66, 3908 (1977); Chem. Phys. Lett. 47, 300 (1977), and references cited therein. Orbital designations are in accordance with the assignments made in the references cited.

${ }^{c}$ The authors cited in the preceding footnote apparently designate the $d$ series as $\left(n d b_{2}\right)^{1} A_{1}$, rather than $\left(n d a_{2}\right)^{1} B_{1}$, and also assign an $n f$ Rydberg series in this spectral interval.

dRecent GVB and IVO calculations of L. B. Harding and W. A. Goddard III, J. Am. Chem. Soc. 99, 677 (1977). Earlier theoretical $\mathrm{H}_{2} \mathrm{CO}$ excitation energies in various approximations are cited in Table IV of the preceding reference. The experimental assignments of the $\left(3 p a_{1}\right)^{1} B_{2}$ and $\left(3 p b_{2}\right)^{1} A_{1}$ states are apparently inadvertently interchanged in their table. See also P. W. Langhoff, S. R. Langhoff, and C. T. Corcoran, J. Chem. Phys. 67, 1722 (1977), for recent CI calculations of discrete transition frequencies and oscillator strengths in $\mathrm{H}_{2} \mathrm{CO}$.

other $(d \text { or } f)^{10}$ Rydberg states of $a_{1}$ type due to lack of appropriate diffuse functions (Table I), and there is little evidence of intense valence transitions into $a_{1} \sigma^{*}$ orbitals. These valence orbitals, in fact, appear in the corresponding photoionization continua, as is discussed further below. In $n a_{2}, n b_{1}$, and $n b_{2}$ final-state symmetries, only single ( $d, p$, and $p$, respectively) Rydberg series are present, and there are intense transitions into $b_{1} \pi^{*}(\mathrm{CO})$ orbitals, according to expectations, but no evidence of strong intravalence transitions into $b_{2} \sigma^{*}(\mathrm{CH}) / \pi_{y}^{*}(\mathrm{CO})$ molecular orbitals. As in the case of $a_{1}$ symmetry, these resonance orbitals, in fact, appear in the photoionization continua of $k b_{2}$ symmetry, a feature of the spectrum discussed in detail below.

\section{1. $2 b_{2}(n)$ excitation (I.P. $\left.=10.9 \mathrm{eV}\right)$}

In Table $\Pi$ are shown the discrete $2 b_{2}-n a_{1}, n b_{2}$, and $n a_{2}$ excitations converging on the ${ }^{2} B_{2}$ ground state in $\mathrm{H}_{2} \mathrm{CO}^{+}$obtained from the IVO calculations, from available experimental assignments, and from other recent theoretical studies. The corresponding continuum photoionization profiles are given in Fig. 2. In each case, the total electronic excitation symmetries are $\left(2 b_{2}\right)^{1} A_{1}$ $\rightarrow\left(n a_{1}\right)^{1} B_{2},\left(n b_{2}\right)^{1} A_{1}$, and $\left(n a_{2}\right)^{1} B_{1}$, corresponding to elec-

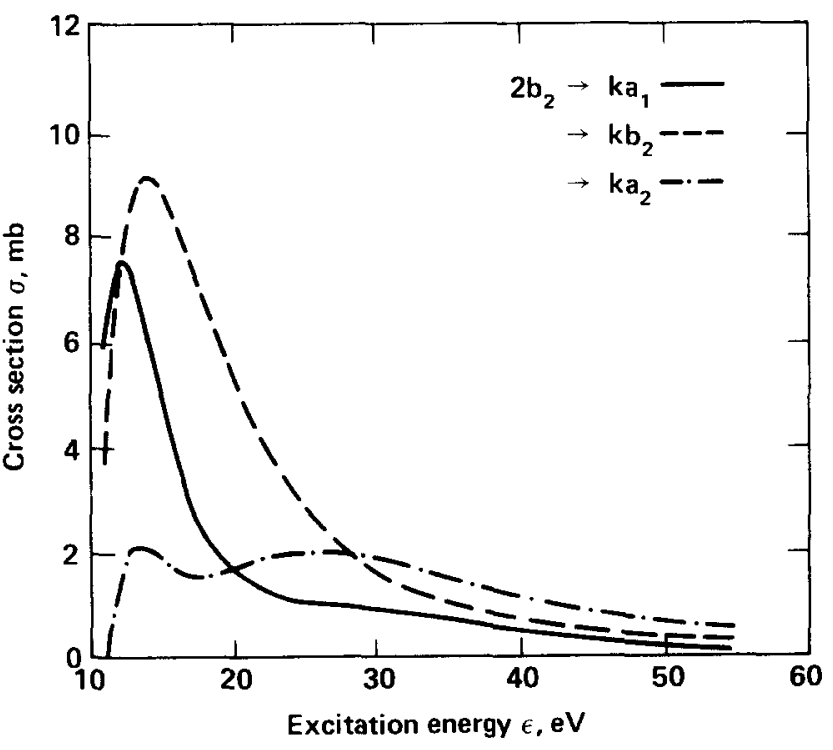

FIG. 2. Improved-virtual-orbital continuum vertical electronic spectra in $\mathrm{H}_{2} \mathrm{CO}$ for $2 b_{2}(n)$ orbital ionization obtained from the S-T technique of Sec. II and discrete-basis set solutions of Eqs. (14), as described in Sec. IV: (-) $2 b_{2} \rightarrow k a_{1} ;(---) 2 b_{2}$ $\rightarrow k b_{2} ;(-\cdots-\cdot-) 2 b_{2} \rightarrow k a_{2}$. 
TABLE III. $1 b_{1}(\pi)$ excitation spectra in $\mathrm{H}_{2} \mathrm{CO}($ I. P. $=14.4 \mathrm{eV})$.

\begin{tabular}{|c|c|c|c|c|c|}
\hline \multicolumn{2}{|c|}{ Present results ${ }^{\mathrm{a}}$} & \multicolumn{2}{|c|}{ Experimental values ${ }^{b, c}$} & \multicolumn{2}{|c|}{ Previous calculations $^{d}$} \\
\hline & $\begin{array}{l}\text { Energy (eV)/ } \\
f \text { number }\end{array}$ & & $\begin{array}{l}\text { Energy (eV)/ } \\
\text { intensity }\end{array}$ & & $\begin{array}{l}\text { Energy }(\mathrm{eV}) / \\
f \text { number }\end{array}$ \\
\hline $\begin{array}{r}{ }^{1} B_{2}\left(1 a_{2}\right) \\
\left(2 a_{2}\right) \\
\left(3 a_{2}\right) \\
\left(4 a_{2}\right) \\
\left(5 a_{2}\right) \\
\left(6 a_{2}\right)\end{array}$ & $\begin{array}{l}12.86 / 0.0150 \\
13.53 / 0.00810 \\
13.84 / 0.00448 \\
14.01 / 0.00263 \\
14.11 / 0.00101 \\
14.23 / \cdots\end{array}$ & $\begin{array}{r}{ }^{1} B_{2}\left(3 d a_{2}\right) \\
\left(4 d a_{2}\right) \\
\left(5 d a_{2}\right) \\
\left(6 d a_{2}\right)\end{array}$ & $\begin{array}{l}12.5 / \cdots \\
13.3 / \cdots \\
13.8 / \cdots \\
14.0 / \cdots \\
\cdots \\
\cdots\end{array}$ & ${ }^{1} B_{2}\left(3 d a_{2}\right)$ & $\begin{array}{l}12.75,12,98 / \cdots, \cdots \\
\ldots \\
\ldots \\
\ldots \\
\ldots \\
\ldots\end{array}$ \\
\hline $\begin{array}{r}1 A_{1}\left(2 b_{1}\right) \\
\left(3 b_{1}\right) \\
\left(4 b_{1}\right) \\
\left(5 b_{1}\right) \\
\left(6 b_{1}\right) \\
\left(7 b_{1}\right) \\
\left(8 b_{1}\right)\end{array}$ & $\begin{array}{l}11.47 / 0.474 \\
12.24 / 0.170 \\
13.26 / 0.0109 \\
13.71 / 0.00304 \\
13.94 / 0.00130 \\
14.07 / 0.00068 \\
14.18 / \cdots\end{array}$ & $\begin{array}{r}{ }^{1} A_{1}\left(2 p b_{1}\right) \\
\left(3 p b_{1}\right) \\
\left(4 p b_{1}\right) \\
\left(5 p b_{1}\right)\end{array}$ & $\begin{array}{l}10.7 / \cdots \\
11.7 / \cdots \\
13.1 / \cdots \\
13.7 / \cdots \\
\cdots \\
\cdots \\
\cdots\end{array}$ & $\begin{array}{c}{ }^{1} A_{1}\left(\pi \rightarrow \pi^{*}\right) \\
\left(3 p b_{1}\right)\end{array}$ & $\begin{array}{l}10.77,11.30 / 0.255, \cdots \\
12.00,12.58 / \cdots, \cdots \\
\cdots \\
\cdots \\
\cdots \\
\cdots \\
\cdots\end{array}$ \\
\hline $\begin{aligned}{ }^{1} B_{1} & \left(6 a_{1}\right) \\
& \left(7 a_{1}\right) \\
& \left(8 a_{1}\right) \\
& \left(9 a_{1}\right) \\
& \left(10 a_{1}\right) \\
& \left(11 a_{1}\right)\end{aligned}$ & $\begin{array}{l}10.99 / 0.0592 \\
11.82 / 0.0189 \\
12.85 / 0.0212 \\
13.15 / 0.0090 \\
13.75 / \cdots \\
14.03 / \cdots\end{array}$ & $\begin{array}{r}{ }^{1} B_{1}\left(3 s a_{1}\right) \\
\left(3 p a_{1}\right) \\
\left(4 s a_{1}\right) \\
\left(4 p a_{1}\right) \\
\left(5 s a_{1}\right)\end{array}$ & $\begin{array}{l}11.1 / \cdots \\
11.7 / \cdots \\
12.9 / \cdots \\
13.1 / \cdots \\
13.6 / \cdots \\
\cdots\end{array}$ & $\begin{aligned}{ }^{1} B_{1} & \left(3 s a_{1}\right) \\
& \left(3 d_{y^{2}} a_{1}\right) \\
& \left(3 d_{z^{2}-x^{2}} a_{1}\right)\end{aligned}$ & $\begin{array}{l}10.73,11.02 / 0.026, \cdots \\
12.58,12.86 / 0.0001, \cdots \\
12.70,12.96 / 0.015, \cdots \\
\cdots \\
\cdots \\
\cdots\end{array}$ \\
\hline
\end{tabular}

${ }^{2} \mathrm{As}$ in Table II.

Tentative spectral assignments of M. J. Weiss et al. (Ref. 5), made primarily on the basis of the observed ioni zation potential and expected quantum defects, and of L. B. Harding and W. A. Goddard III (Ref. 26), made on basis of unpublished data of $A$. Chutjian. The values cited by the former authors (Ref. 5) have been shifted uniformly by $\sim 0.3 \mathrm{eV}$ to adjust for the use of an adiabatic ionization potential.

'The assignment of the $\pi \rightarrow \pi^{*}$ transition at $10.7 \mathrm{eV}$ by L. B. Harding and W. A. Goddard (Ref. 26) must be regarded as tentative, since the measured spectra (Refs. 4 and 5) do not indicate the presence there of a strong transition.

${ }^{\mathrm{d}} \mathrm{As}$ in Table II.

TABLE IV. $5 a_{1}(\sigma), 1 b_{2}$, and $4 a_{1}$ excitation spectra in $\mathrm{H}_{2} \mathrm{CO}{ }^{\text {a }}$

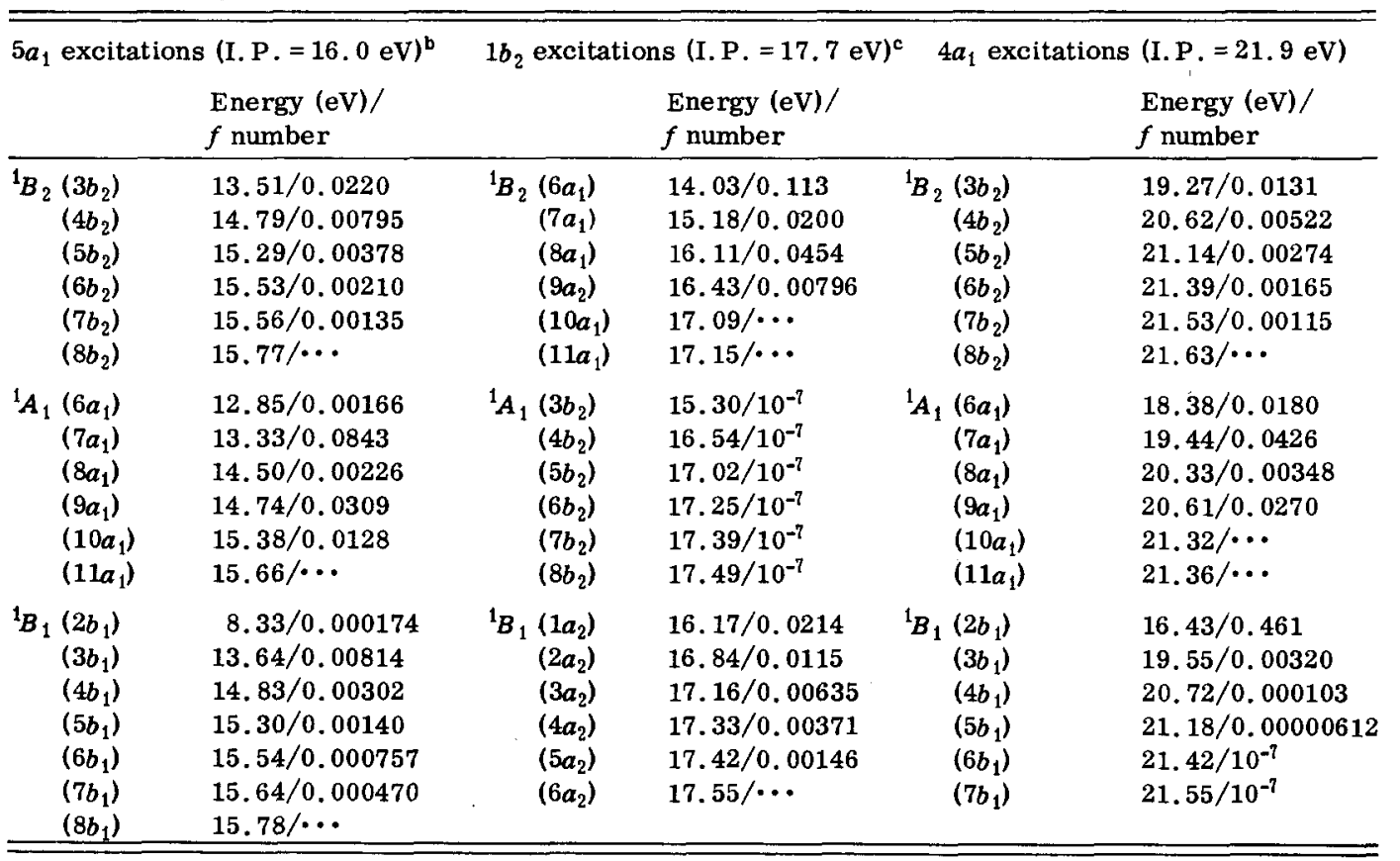

${ }^{2} \mathrm{As}$ in Table II.

${ }^{\text {T The valence }} 5 a_{1} \rightarrow 2 b_{1}$ transition appears in the measured spectra (Refs. 4 and 5 ) at $\sim 8.7$ to $9.2 \mathrm{eV}$ as a series of weak broad bands. Moreover, tentative spectral assignments (Ref. 5) of the $n s$ (12.6, $14.5,15.1 \mathrm{eV}), n p(13.1,14.6,15.2 \mathrm{eV})$, and $n d(13.9,15.0,15.3 \mathrm{eV})$ Rydberg series converging on the $\left(5 a_{1}^{-1}\right)^{2} A_{1}$ ionic state are in general accord with the present IVO results.

'The experimental ionization potential of $16.8 \mathrm{eV}$ (Ref. 2) is apparently uncertain at present and consequently the $17.7 \mathrm{eV}$ Koopmans value is employed in the present development. 
tric fields having $y$ (in plane), $z$ (in plane), and $x$ (out of plane) polarizations, respectively.

The calculated $2 b_{2}-n a_{1}$ excitations are seen to contain two distinct medium-intensity Rydberg series, which are in generally good accord with the experimental assignments shown in Table II for the $n s a_{1}$ and $n p a_{1}$ series. ${ }^{4-11}$ In addition, the calculated resonance $f$ numbers for the two series are in general agreement with the two available measured values, ${ }^{4,5}$ and with recent IVO and GVB calculations. ${ }^{26}$ Moreover, the effective principal quantum numbers of these resonance transitions (2.0 and 2.3) suggest Rydberg character. ${ }^{48}$ Since many of the earlier ab initio calculations of the $\left(3 s a_{1}\right)^{1} B_{2}$ and $\left(3 p a_{1}\right)^{1} B_{2}$ states in formaldehyde are cited in the previous references, the corresponding energies and strengths are not reproduced here.

It is of considerable interest to note the absence of a strong intravalence $2 b_{2}-a_{1} \sigma^{*}$ transition in the $\mathrm{H}_{2} \mathrm{CO}$-discrete spectrum of Table $\Pi$. Referring to Fig. 2, however, which gives the corresponding $2 b_{2} \rightarrow k a_{1}$ photoionization profile, it is evident that compact valencelike $a_{1} \sigma^{*}$ orbitals appear in the continuum at $\sim 12 \mathrm{eV}$, similar to the situation in the previously studied CO spectrum. ${ }^{31}$ Since the $2 b_{2}(n)$ orbital is $\mathrm{O}\left(2 p_{y}\right)$ nonbonding, weakly $\sigma(\mathrm{CH})$ bonding, and weaker still $\pi_{y}(\mathrm{CO})$ bonding, ${ }^{47}$ resonance transitions into both $a_{1} \sigma^{*}(\mathrm{CO})$ and $a_{1} \sigma^{*}(\mathrm{CH})$ valence orbitals are possible, although the $a_{1} \sigma^{*}(\mathrm{CO})$ component presumably dominates. ${ }^{31}$ The large contribution from $2 b_{2} \rightarrow k a_{1}$ excitation in the threshold region shown in Fig. 2 is also observed in recent CI S-T calculations of the ${ }^{1} A_{1} \rightarrow{ }^{1} B_{2}$ cross section. ${ }^{28}$ In addition to the resonance feature at $\sim 12 \mathrm{eV}$ in the $2 b_{2} \rightarrow k a_{1}$ profile, continuum excitations of background or Rydberg $2 b_{2} \rightarrow k s a_{1}$ and $k p a_{1}$ type also contribute to the photoionization cross section for this channel. However, since the $2 b_{2} \rightarrow n s a_{1}$ Rydberg intensities form a decreasing Stieltjes histogram, ${ }^{33}$ it is likely that the $2 b_{2} \rightarrow k p a_{1}$ excitations provide the major Rydberg contribution to the $2 b_{2} \rightarrow k a_{1}$ photoionization cross section. Unfortunately, it is not possible to disentangle the two contributions on the basis of the present calculations.

The calculated IVO $2 b_{2} \rightarrow n b_{2}$ transitions shown in Table $\Pi$ evidently correspond to a single excitation series of monotonically decreasing $f$ values in excellent accord with the experimentally assigned $2 b_{2} \rightarrow n p b_{2}$ series. $6,7,10$ Moreover, the resonance $f$ number is in general agreement with the available measured value, ${ }^{4,5}$ and with the results of recent GVB and IVO calculations. ${ }^{26}$ An effective principal quantum number of 2.3 indicates the Rydberg character of this transition. ${ }^{48}$ There is no evidence of a separate series of $n d b_{2}$ excitations in the calculated IVO spectrum, however, apparently as a consequence of the lack of sufficient numbers of diffuse $d$ functions in the basis employed (Table I). The $2 b_{2} \rightarrow 3 d b_{2}$ excitation predicted in previous calculations is apparently quite weak, ${ }^{26}$ however, and consequently the diffuse $d$ function deficiency in the $b_{2}$ spectrum should not affect the present results significantly.

Evidently, there is no intense $2 b_{2} \rightarrow b_{2} \sigma^{*}(\mathrm{CH}) / \pi_{y}^{*}(\mathrm{CO})$ intravalence transition present in the discrete spectra of Table II, in spite of the previously indicated expectations. However, the $2 b_{2} \rightarrow k b_{2}$ continuum profile of Fig. 2 exhibits a large resonancelike peak at $\sim 14 \mathrm{eV}$ which can be attributed to the presence of $b_{2} \pi_{y}^{*}(\mathrm{CO})$ valencelike orbitals in the photoionization continuum, and presumably corresponds to an $n-\pi_{y}^{*}$ transition in the $\mathrm{CO}$ bond. This result is in excellent accord with the recent CI S-T calculations, ${ }^{28}$ which indicate large $2 b_{2}-k b_{2}$ excitations at $\sim 14 \mathrm{eV}$ interacting weakly with $1 b_{1} \rightarrow n b_{1}$ Rydberg excitations in the ${ }^{1} A_{1} \rightarrow{ }^{1} A_{1} \mathrm{H}_{2} \mathrm{CO}$ cross section. The latter interaction, of course, is not explicitly included in the present IVO calculations.

The calculated $2 b_{2} \rightarrow n a_{2}$ transitions of Table II are evidently very weak, and correspond to a single excitation series of monotonically decreasing $f$ numbers. Although the transition energies are in general accord with the recently assigned $n d$ series, the experimental series is evidently regarded as $n d b_{2}$, rather than $n d a_{2}{ }^{10}$ Moreover, the relatively weak resonance transition intensity in this case suggests that pre-Rydberg $3 d$ orbitals $^{49}$ possibly appear in the $2 b_{2}(n) \rightarrow k a_{2}$ photoionization continuum, rather than in the discrete spectral region. Indeed, although the $2 b_{2} \rightarrow k a_{2}$ profile of Fig. 2 is seen to be vanishingly small at threshold and generally weak, a resonancelike feature is evidently present at $\sim 14 \mathrm{eV}$, with a secondary broad structure at $\sim 27 \mathrm{eV}$. A similar resonancelike $1 \pi \rightarrow 3 d \delta$ transition appears in the photoionization continuum of CO. ${ }^{31}$ The generally weak contribution of $2 b_{2}-k a_{2}$ excitations to the photoionization cross section observed in the present IVO $\mathrm{S}-\mathrm{T}$ calculations is in accord with the previously reported CI S-T results, which indicate small contributions from such excitations to the ${ }^{1} A_{1}-{ }^{1} B_{1}$ cross section. ${ }^{28}$ The lack of $2 b_{2} \rightarrow k a_{2}$ excitations in the previous CI S-T calculations is also, however, possibly a partial consequence of the lack of diffuse $d$ functions in the basis set employed.

\section{2. $1 b_{1}(\pi)$ excitation (I.P. $\left.=14.4 \mathrm{eV}\right)$}

In Table III are shown the discrete $1 b_{1} \rightarrow n a_{2}, n b_{1}$, and $n a_{1}$ IVO excitations converging on the $\left(1 b_{1}^{-1}\right)^{2} B_{1}$ state in $\mathrm{H}_{2} \mathrm{CO}^{+}$, with corresponding continuum profiles shown in Fig. 3. Also shown in the table are experimental assignments and the results of previous calculations. The total electronic transition symmetries are $\left(1 b_{1}\right)^{1} A_{1}$ $\rightarrow\left(n a_{2}\right)^{1} B_{2},\left(n b_{1}\right)^{1} A_{1}$, and $\left(n a_{1}\right)^{1} B_{1}$, corresponding to $y, z$, and $x$ polarizations, respectively. It is perhaps appropriate to note that although the transitions of Table III are discrete in the sense of falling below the $1 b_{1}(\pi)$ ionization threshold $(14.4 \mathrm{eV})$, they appear in the continua of the previously described $2 b_{2}$ excitations (see Fig. 1), and thus can undergo autoionization. Linewidths due to this mechanism are not treated explicitly in the present investigation.

The calculated $1 b_{1}-n a_{2}$ excitations shown in Table III evidently comprise a single $n d a_{2}$ series of decreasing $f$ numbers, having transition energies in good accord with the available experimental assignments and previously reported calculations. ${ }^{5,26}$ However, the experimental assignments are tentative, based primarily on the known ionization potentials and expected quantum defects, and 


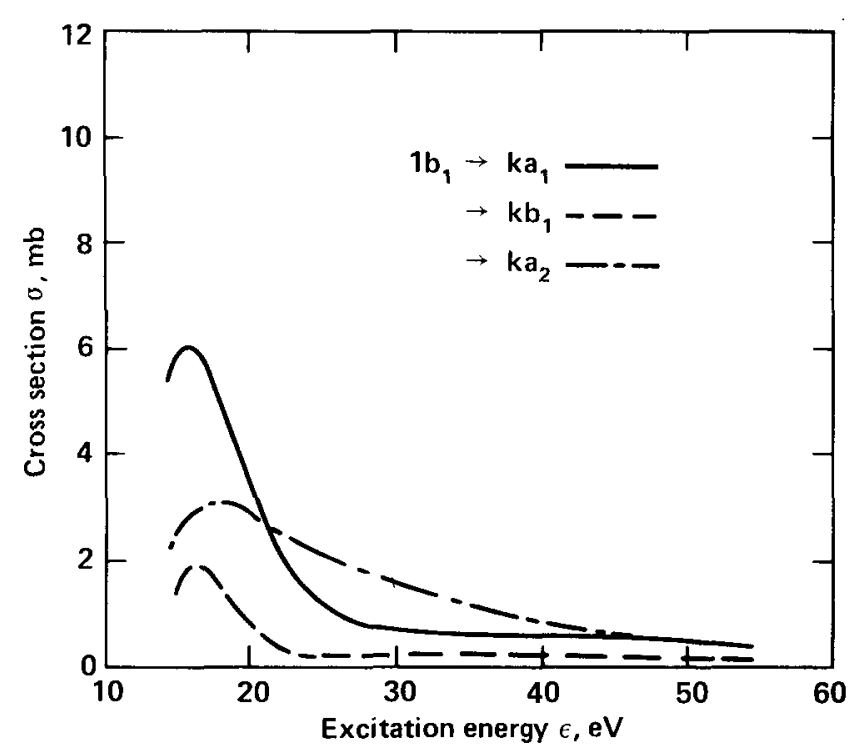

FIG. 3. As in Fig. 2, for $1 b_{1}(\pi)$ orbital ionization: (-) $1 b_{1}$ $\rightarrow k a_{1} ;(---) 1 b_{1} \rightarrow k b_{1}$; (-・--.-) $1 b_{1} \rightarrow k a_{2}$.

the predicted medium intensity $1 b_{1} \rightarrow 1 a_{2}$ resonance excitation is apparently not present in the observed spectra. ${ }^{5}$ Presumably, the situation can be clarified by detailed autoionization lineshape calculations. Although there are no intense intravalence transitions in the $1 b_{1}$ - $n a_{2}$ discrete excitations of Table III, in accordance with expectations, Fig. 3 indicates the presence of a weak, broad resonancelike feature in the $1 b_{1}-k a_{2}$ photoionization profile at $\sim 17 \mathrm{eV}$. This structure is similar to that appearing in the $1 \pi-k \delta$ photoionization continuum in the previously studied CO cross section, ${ }^{31}$ and can presumably be attributed to the presence of pre-Rydberg $3 d$ orbitals in the $k a_{2}$ photoionization continuum. ${ }^{49}$ This view is supported by the moderate intensity of the calculated resonance transition in this series, which is perhaps too small to warrent a $3 d a_{2}$ designation. The previously reported CI S-T calculations indicate the presence of double excitations in the ${ }^{1} A_{1}-{ }^{1} B_{2}$ cross section at $\sim 17 \mathrm{eV}$, which may correspond in these previous calculations to $\left(1 b_{1} \rightarrow k a_{2}\right)$-like transitions, due to $d a_{2}$ basis set deficiency. ${ }^{28}$

The calculated $1 b_{1} \rightarrow n b_{1}$ excitations evidently correspond to a single $n p b_{1}$ series of decreasing $f$ numbers, with the expected very intense resonance $\pi-\pi^{*}$ transi tion, in good agreement with the previous calculations. ${ }^{26}$ The experimental assignment at $10.7 \mathrm{eV}$ is somewhat suspect, ${ }^{26}$ however, since the absorption and electron impact-excitation measurements do not show significant intensity at this energy. ${ }^{4,5}$ Rather, it is reasonable to suggest that the $\pi \rightarrow \pi^{*}$ transition in $\mathrm{H}_{2} \mathrm{CO}$ corresponds to a strong broad feature in the measured spectrum at $\sim 13.5 \mathrm{eV},{ }^{8}$ located there by vibronic autoionization of the $\left(\pi \rightarrow \pi^{*}\right)^{1} A_{1}$ state which has a potential curve repulsive in $\mathrm{C}-\mathrm{O}$ stretch ${ }^{14}$ and apparently favors a nonplanar configuration. ${ }^{7,50}$ Moreover, although the tentative experimentally assigned Rydberg positions are in general agreement with the IVO calculations, the strong predicted intensities are not observed in the measured spectra. ${ }^{4,5}$ Again, autoionization line shape cal- culations are required to clarify the situation. Because much of the $f$ sum rule is taken up by the discrete transitions in the calculated spectrum, the $1 b_{1} \rightarrow k b_{1}$ photoionization continuum of Fig. 3 is generally weak, although the presence of a small maximum at $\sim 16 \mathrm{eV}$ is in accord with the CI S-T calculations, ${ }^{2 B}$ and similar to the $1 \pi-k \pi$ profile in $\mathrm{CO} .^{31}$

Evidently, both $1 b_{1} \rightarrow n s a_{1}$ and $n p a_{1}$ excitations are present in the IVO $n a_{1}$ series of Table III. The calculated positions are in agreement with the experimental assignments and previous calculations, although the predicted medium-intensity Rydberg transitions are not apparent in the measured spectra. ${ }^{4,5}$ There is no evidence of intense intravalence transitions in the $1 b_{1}$ $-n s a_{1}$ discrete excitation series, and the lowest effective principal quantum number is $\sim 2.0{ }^{48}$ However, referring to Fig. 3 , the $1 b_{1}-k a_{1}$ continuum cross section has a strong resonancelike feature at $\sim 16 \mathrm{eV}$ which can be attributed to transition into the valencelike $a_{1} \sigma^{*}(\mathrm{CO})$ orbital responsible for a similar resonance in the $2 b_{2} \rightarrow k a_{1}$ profile (Fig. 2) described above. Strong $1 b_{1} \rightarrow k a_{1}$ excitations at $\sim 16 \mathrm{eV}$ also contribute to the previously reported ${ }^{1} A_{1} \rightarrow{ }^{1} B_{1}$ CI S-T spectrum, ${ }^{28}$ providing independent evidence for the existence of this resonance.

\section{3. $5 a_{1}(\sigma)$ excitation (I.P. $\left.=16.0 \mathrm{eV}\right)$}

In Table IV are shown the discrete $5 a_{1} \rightarrow n b_{2}, n a_{1}$, and $n b_{1}$ excitations converging on the $\left(5 a_{1}^{-1}\right)^{2} A_{1}$ state in $\mathrm{H}_{2} \mathrm{CO}^{+}$, with corresponding continuum profiles shown in Fig. 4. The total electronic symmetries are $\left(5 a_{1}\right)^{1} A_{1} \rightarrow\left(n b_{2}\right)^{1} B_{2}$, $\left(n a_{1}\right)^{1} A_{1}$, and $\left(n b_{1}\right)^{1} B_{1}$, corresponding to $y, z$, and $x$ polarizations, respectively.

The $5 a_{1}-n b_{2}$ excitations of Table IV evidently corre spond to a single $n p b_{2}$ series of decreasing $f$ numbers having positions in good agreement with the tentative expe rimental $n p(13.1,14.6,15.2 \mathrm{eV})$ Rydberg series assignment. ${ }^{5}$ As in the case of the $2 b_{2} \rightarrow n b_{2}$ excitations

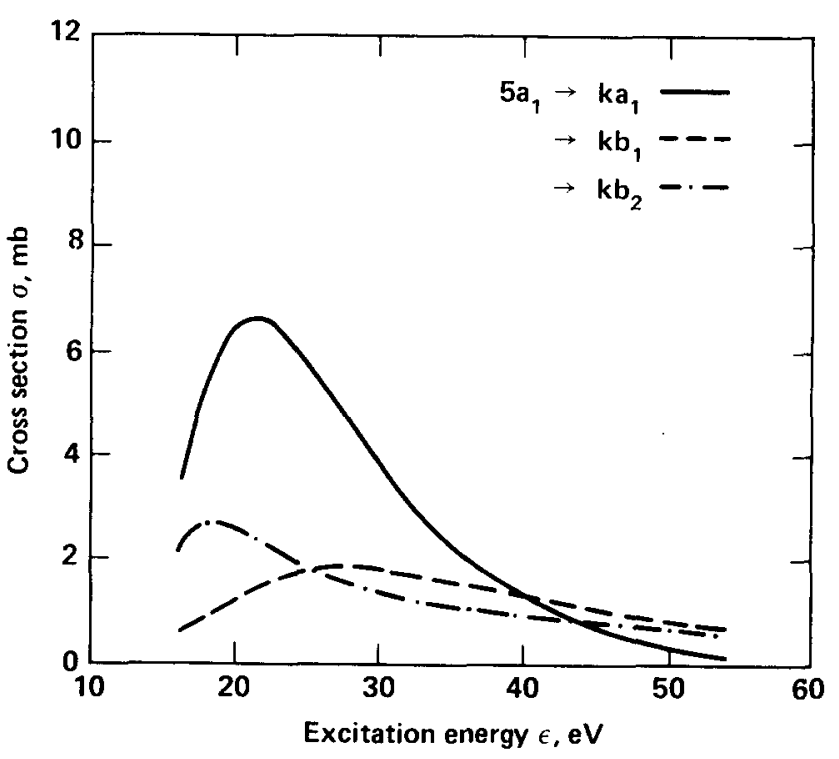

FIG. 4. As in Fig. 2, for $5 a_{1}(\sigma)$ orbital ionization: (-) $5 a_{1}$ $\rightarrow k a_{1} ;(---) 5 a_{1} \rightarrow k b_{1} ;(-.-.--) 5 a_{1} \rightarrow k b_{2}$. 
(Table II), there is no evidence of a separate $5 a_{1} \rightarrow n d b_{2}$ series, presumably as a consequence of the lack of diffuse $d$ orbitals in the $b_{2}$ basis (Table I). In addition, there is no intense resonancelike transition into a $b_{2} \sigma^{*}(\mathrm{CH}) / \pi_{y}^{*}(\mathrm{CO})$ valence orbital present in the $5 a_{1}$ $-n b_{2}$ series of Table IV. Rather, a weak resonance appears in the $5 a_{1}-k b_{2}$ photoionization continuum of Fig. 4, suggesting again, as in Fig. 2, that valencelike $b_{2} \sigma^{*}(\mathrm{CH}) / \pi_{y}^{*}(\mathrm{CO})$ virtual orbitals appear in the photoionization continuum. The general positioning of the IVO resonance peak at $\sim 18 \mathrm{eV}$ in this case is also in very good agreement with the previously reported CI S-T calculations of ${ }^{1} A_{1} \rightarrow{ }^{1} B_{2}$ excitations. ${ }^{28}$

The $5 a_{1}-n a_{1}$ excitations of Table IV evidently contain distinct $n s a_{1}$ and $n p a_{1}$ series, which apparently correspond to $f$ numbers of increasing and decreasing intensity, respectively, and have positions in general accord with the tentative experimental $n s(12.6,14.5$, $15.1 \mathrm{eV})$ and $n p(13.1,14.6,15.2 \mathrm{eV})$ Rydberg series assignments, respectively. ${ }^{5}$ Although the intensities of these series require further clarification, it is certain that strong valencelike $5 a_{1}(\sigma)-a_{1} \sigma^{*}(\mathrm{CO})$ and $a_{1} \sigma(\mathrm{CH})$ transitions are not present in either series. Rather, referring to Fig. 4, a very intense and broad resonance feature is evident in the $5 a_{1} \rightarrow k a_{1}$ photoionization continuum, which in this case can be attributed primarily to the $a_{1} \sigma^{*}(\mathrm{CO})$ valence-orbital contribution. Since the continuum transition in this case is presumably $\sigma \rightarrow \sigma^{*}$ in the $\mathrm{C}-\mathrm{O}$ bond, it is particularly strong, as in the corresponding $5 \sigma-k \sigma *$ resonance in $\mathrm{CO} .{ }^{31}$ This very broad feature is also in excellent accord with the previously reported CI S-T results, which show strong $5 a_{1} \rightarrow k a_{1}$ excitation in the ${ }^{1} A_{1}-{ }^{1} A_{1}$ cross section. ${ }^{28}$

The $5 a_{1}-n b_{1}$ excitation series of Table IV evidently contains a weak low -lying $5 a_{1}-b_{1} \pi^{*}$ intravalence transitions, in accord with expectations and with experiment, ${ }^{4}$ as well as a $5 a_{1}-n p b_{1}$ Rydberg series having positions in accord with the tentative $n p(13.1,14.6,15.2 \mathrm{eV})$ experimental assignment. ${ }^{5}$ The very low intensity of the valence transition is clarified by noting that the $b_{1} \pi^{*}$ orbital has a node in the $y z$ plane, including the $\mathrm{C}-\mathrm{O}$ axis along which the $5 a_{1}$ has maximum density. The corresponding continuum $5 a_{1}-k b_{1}$ photoionization profile of Fig. 4 is weak but has a broad shoulder extending to high energy, in very good qualitative agreement with the CI S-T ${ }^{1} A_{1}-{ }^{1} B_{1}$ cross section. ${ }^{28}$

\section{4. $1 b_{2}$ excitation (I.P. $\left.=17.7\right)$}

In Table IV are shown the disc rete $1 b_{2}-n a_{1}, n b_{2}$, and $n a_{2}$ excitations converging on the $\left(1 b_{2}^{-1}\right)^{2} B_{2}^{*}$ state in $\mathrm{H}_{2} \mathrm{CO}^{+}$, with the corresponding continuum profiles shown in Fig. 5. The total electronic transition symmetries are identical to those of the $2 b_{2}$ excitations discussed above (Sec. V.A.1) .

Referring to Table IV, both $1 b_{2}-n s a_{1}$ and $n p a_{1}$ transitions are evident in the $n a_{1}$ series. The resonance in the $n s a_{1}$ series $\left(n^{*} \sim 1.9\right)$ is the second strongest Ryd berg transition of Tables II to IV, presumably as a consequence of the large spatial extent of the $1 b_{2}$ orbital, which has charge density in both $\mathrm{C}-\mathrm{O}$ and $\mathrm{C}-\mathrm{H}$ bonds. ${ }^{47}$ As expected, the $1 b_{2} \rightarrow k a_{1}$ photoionization profile of

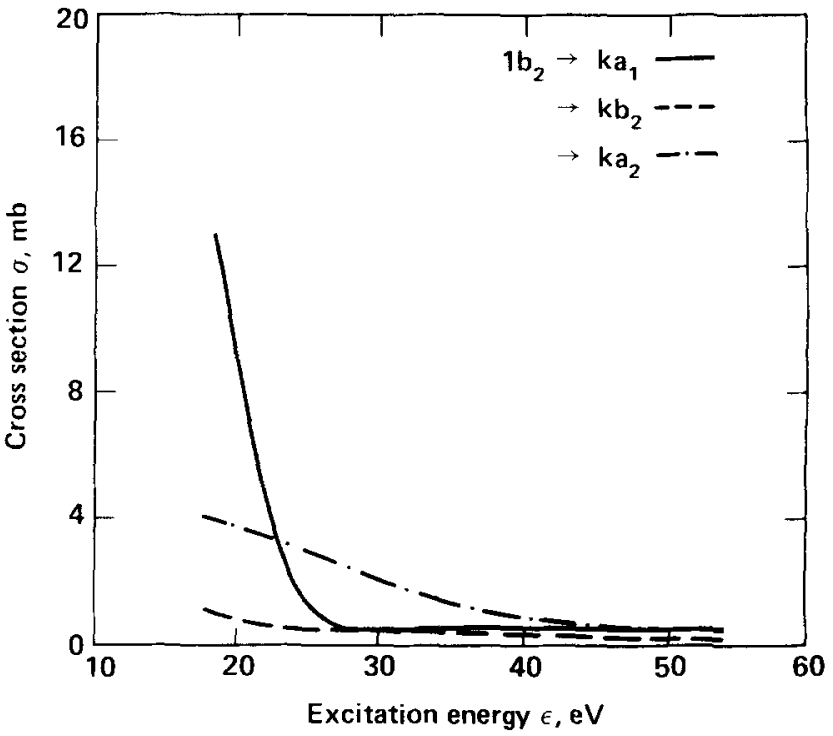

FIG. 5. As in Fig. 2, for $1 b_{2}$ orbital ionization; $(-) 1 b_{2} \rightarrow k a_{1}$; $(---) 1 b_{2} \rightarrow k b_{2} ;(-\cdot-\cdot-) 1 b_{2} \rightarrow k a_{2}$.

Fig. 5 contains an $a_{1} \sigma^{*}(\mathrm{CO})$ resonance feature at $\sim 18 \mathrm{eV}$, in qualitative accord with the previously reported CI S-T results. ${ }^{28}$

The very low intensity of the $1 b_{2} \rightarrow n p b_{2}$ Rydberg series can be regarded as a consequence of the largely $2 p_{y} \rightarrow n p_{y}$ nature of these transitions located at the c.m. By contrast, the $2 b_{2}(n)-n b_{2}$ Rydberg series (Table II) is of medium intensity, presumably since the $2 b_{2}$ orbital is located primarily at the $\mathrm{O}$ end of the molecule. In spite of the fact that the $1 b_{2}$ orbital contains $\sigma(\mathrm{CH})$ and $\pi_{y}(\mathrm{CO})$ bonding components, there is no evidence of a resonance transition in the $1 b_{2}-k b_{2}$ photoionization continuum of Fig. 5, which is seen to be quite weak.

The $1 b_{2}-n d a_{2}$ Rydberg series of Table IV apparently contains a $1 a_{2}$ resonance transition of only moderate intensity. Consequently, the weak, broad structure in the $1 b_{2} \rightarrow k a_{2}$ photoionization cross section of Fig. 5 can be attributed to the presence of pre-Rydberg $3 d a_{2}$ orbitals in the continuum, ${ }^{49}$ in accord with the results of Figs. 2 and 3 . The previously reported CI S-T results are $d a_{2}$ basis deficient and consequently do not contain these features. ${ }^{28}$

\section{5. $4 a_{1}$ excitation (I.P. $\left.=21.9 \mathrm{eV}\right)$}

In Table IV are shown the discrete $4 a_{1} \rightarrow n b_{2}, n a_{1}$, and $n b_{1}$ excitations conve rging on the $\left(4 a_{1}^{-1}\right)^{2} A_{1}^{*}$ state in $\mathrm{H}_{2} \mathrm{CO}^{+}$, with the corresponding continuum profiles shown in Fig. 6. The total electronic transition symmetries are identical to those of the $5 a_{1}$ excitations described above (Sec.V.A. 3).

The $4 a_{1} \rightarrow n b_{2}$ series is similar to the $5 a_{1} \rightarrow n b_{2}$ excitations of Table IV in that both correspond to $n p b_{2}$ excitations of monotonically decreasing intensity. Similarly, there are both $n s a_{1}$ and $n p a_{1}$ excitations of alternating intensity in the $4 a_{1}-n a_{1}$ series. The intense $4 a_{1} \rightarrow \pi^{*}$ feature at $\sim 16 \mathrm{eV}$ also appears in the previously reported CI S-T calculations. ${ }^{28}$ Although this intense 
$\sigma \rightarrow \pi^{*}$ transition dominates the $4 a_{1} \rightarrow n b_{1}$ series, the corresponding $5 a_{1}-\pi^{*}$ transition in Table IV is evidently weak. Since the $4 a_{1}$ canonical orbital has a large O nonbonding character, whereas the $5 a_{1}$ orbital has maximum density in the $\mathrm{C}-\mathrm{O}$ bond, the intense $4 a_{1}$ $-2 b_{1}\left(\pi^{*}\right)$ but weak $5 a_{1}-2 b_{1}\left(\pi^{*}\right)$ features are in general accord with expectation. ${ }^{24,47}$

The continuum profiles shown in Fig. 6 for $4 a_{1} \rightarrow k b_{2}$, $k a_{1}$, and $k b_{1}$ excitation are evidently quite weak, and the $k b_{2}$ and $k a_{1}$ contributions do not contain the resonances common in the outer - orbital cross sections.

\section{B. Photoionization resonances}

The resonance features appearing in the photoionization cross sections of Figs. 2-6 warrant additional discussion. In order to clarify their natures, the spatial distributions of the final-state resonance orbitals in each case can be unambiguously established by examining the pseudospectral functions contained in the appropriate excitation-energy interval. In order to accomplish such an analysis, the very-large-basis-set IVO orbitals determined in the present studies must be exhibited in suitable graphical forms. This constitutes a major data handling problem which is undertaken in a separate study. In the present report, it is sufficient to infer and describe the natures of the final-state resonance orbitals qualitatively, making use only of the photoionization cross sections of Figs. 2-6 and an understanding of the virtual orbitals anticipated on basis of valence calculations. ${ }^{24,47,51,52}$

In Fig. 7 are shown the four cross sections corresponding to $k a_{1}$ final states in which $a_{1} \sigma *(\mathrm{CO})$ or $a_{1} \sigma(\mathrm{CH})$ resonances occur. Although a resonancelike structure appears in each of the channel cross sections of Fig. 7, there is apparently no evidence of a resonance in the $4 a_{1}-k a_{1}$ cross section (Fig. 6). Since the $4 a_{1}$ orbital is $\mathrm{CH} \sigma$ bonding and $\mathrm{O}$ nonbonding, but includes very little $\mathrm{CO} \sigma$ bonding character, ${ }^{47}$ it is reasonable to suggest

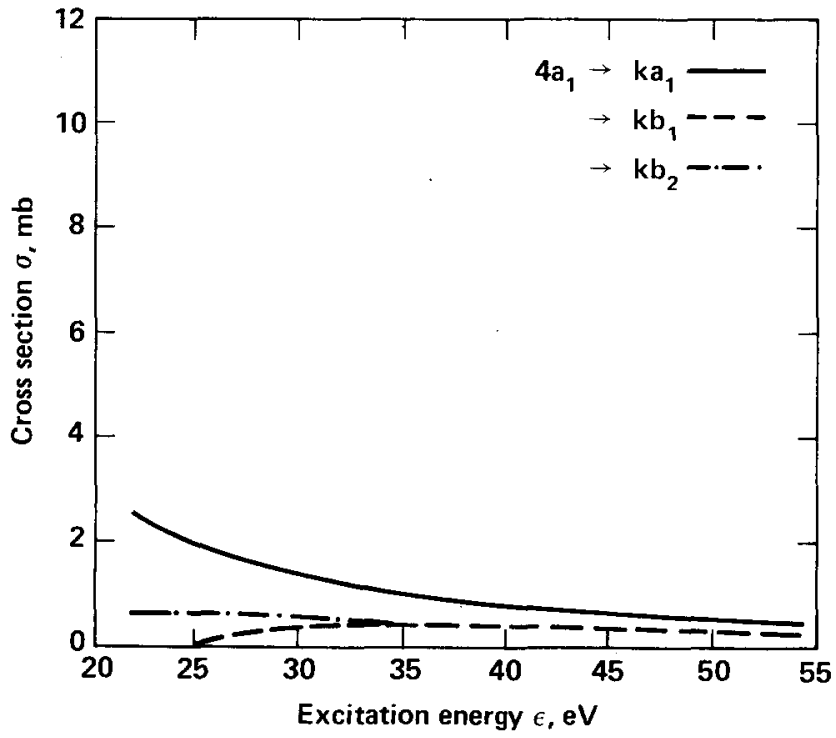

FIG. 6. As in Fig. 2, for $4 a_{1}$ orbital ionization: (-) $4 a_{1} \rightarrow k a_{1}$; $(---) 4 a_{1} \rightarrow k b_{1} ;(-----) 4 a_{1}-k b_{2}$.

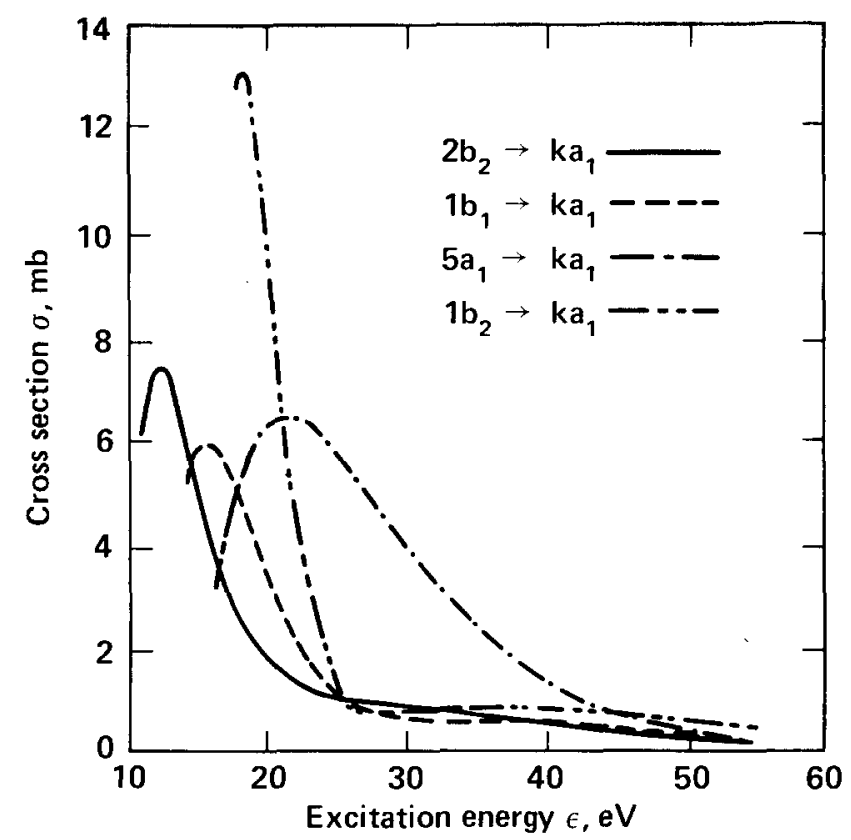

FIG. 7. Improved-virtual-orbital vertical electronic photoionization cross sections having $k a_{1}$ final-state resonances: (-) $2 b_{2} \rightarrow k a_{1} ;(---) 1 b_{1} \rightarrow k a_{1} ;(-\cdots-\circ) 5 a_{1} \rightarrow k a_{1}$ $(-\cdots-\cdots-\cdots) ,1 b_{2} \rightarrow k a_{1}$.

that the important resonance orbital in the final state $k a_{1}$ profile in each case can be regarded as $a_{1} \sigma^{*}(\mathrm{CO}) .^{24}$ When this view is adopted, as it is implicitly in some of the foregoing text, the intensities of the four cross sections shown in Fig. 7 are qualitatively clarified. In particular, the very broad and strong $5 a_{1} \rightarrow k a_{1}$ shape resonance is seen to correspond to a $\sigma \rightarrow \sigma^{*}$ transition in the $\mathrm{CO}$ bond, as indicated above. Similarly, the $2 b_{2}$, $1 b_{1}$, and $1 b_{2} \rightarrow k a_{1}$ resonances can be attributed to $\pi \rightarrow \sigma^{*}$ transitions involving the $\pi$ and pseudo- $\pi$ components of the $1 b_{1}$ and $2 b_{2}, 1 b_{2}$ orbitals, respectively, ${ }^{47}$ and the final-state $a_{1} \sigma^{*}(\mathrm{CO})$ orbital.

In Fig. 8 are shown the two photoionization cross

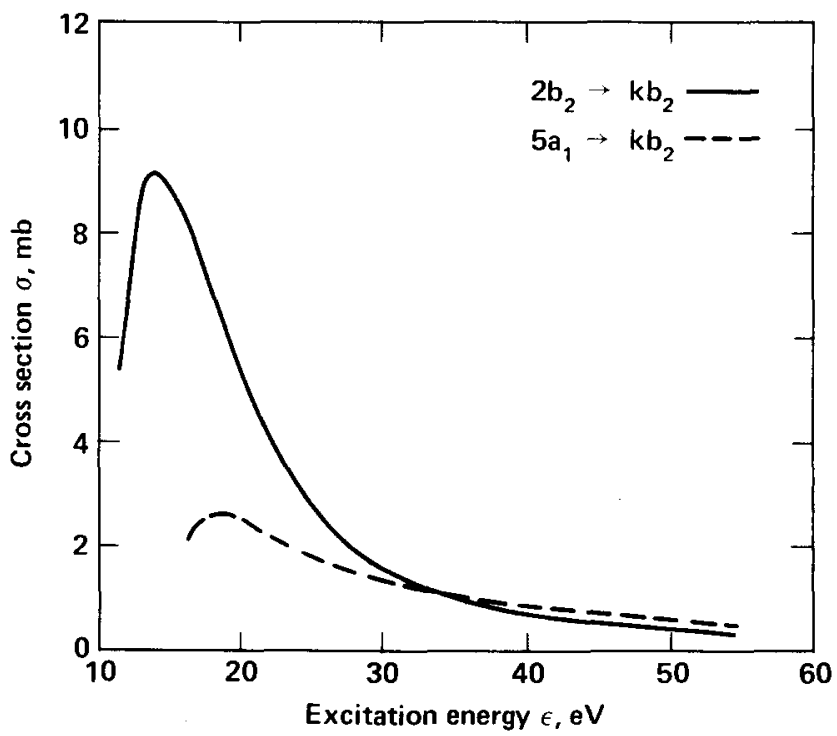

FIG. 8. As in Fig. 7, for $k b_{2}$ final-state resonances; (-) $2 b_{2} \rightarrow k b_{2}$; (---) $5 a_{1} \rightarrow k b_{2}$. 


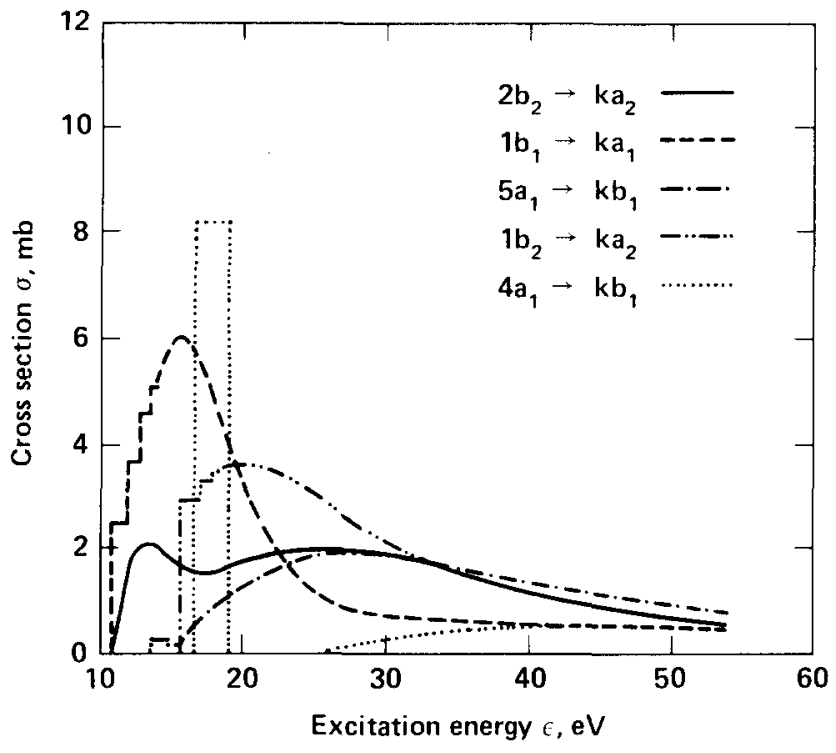

FIG. 9. Individual vertical electronic IVO excitations contributing to the photoionization cross section in $\mathrm{H}_{2} \mathrm{CO}$ for ${ }^{1} A_{1} \rightarrow{ }^{1} B_{1}$ transitions, constructed from Figs. $2-6$ and Tables II-IV: (-) $2 b_{2} \rightarrow a_{2} ;(---) 1 b_{1} \rightarrow a_{1} ;(-\cdot-\cdots,-) 5 a_{1} \rightarrow b_{1} ;(-\cdots, \cdots-\cdots)$ $1 b_{2} \rightarrow a_{2} ;(\cdots \cdots) 4 a_{1} \rightarrow b_{1}$; the discrete or autoionizing transitions are represented in the so-called Stieltjes sense (Ref. 33).

sections that contain resonances in the final $k b_{2} \mathrm{sym}-$ metry. Evidently, resonances appear in the $2 b_{2}-k b_{2}$ and $5 a_{1}-k b_{2}$ channels, but not in the $1 b_{2}-k b_{2}$ (Fig. 5) and $4 a_{1}-k b_{2}$ (Fig. 6) cross sections. This situation is clarified by regarding the final state $k b_{2}$ resonance as primarily $b_{2} \pi_{y}^{*}(\mathrm{CO})$ in character. ${ }^{24}$ When this view is adopted, the resonances of Fig. 8 correspond to $n \rightarrow \pi_{y}^{*}$ and $\sigma \rightarrow \pi_{y}^{*}$ transitions, respectively, and the absence of similar structures in the $1 b_{2} \rightarrow k b_{2}$ and $4 a_{1} \rightarrow k b_{2}$ channels is attributed to the low charge densities of these

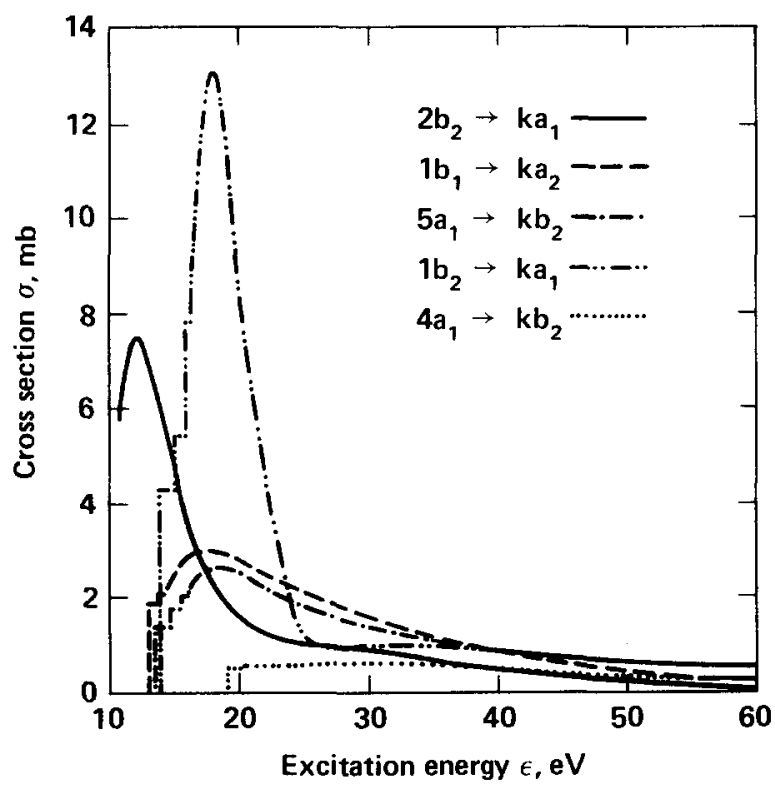

FIG. 10. As in Fig. 9, for ${ }^{1} A_{1} \rightarrow{ }^{1} B_{2}$ transitions in $\mathrm{H}_{2} \mathrm{CO}$ : (-) $2 b_{2} \rightarrow a_{1} ;(---) 1 b_{1} \rightarrow a_{2} ;(-\cdot-\cdot-\cdot) 5 a_{1} \rightarrow b_{2} ;(-\cdots-\cdots-\cdots-)$ $1 b_{2} \rightarrow a_{1} ;(\cdots) 4 a_{1} \rightarrow b_{2}$.

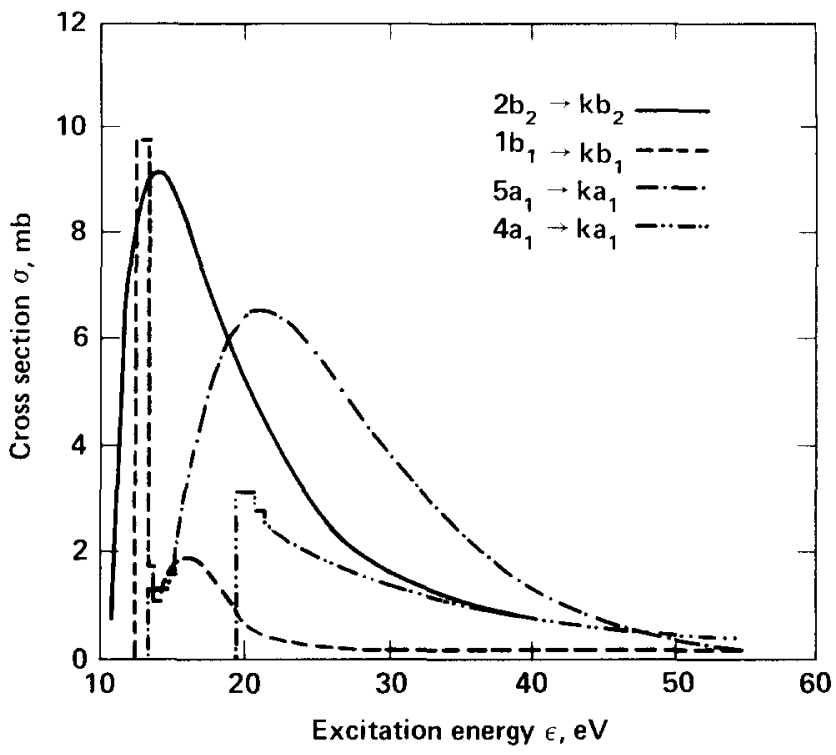

FIG. 11. As in Fig. 9, for ${ }^{1} A_{1} \rightarrow{ }^{1} A_{1}$ transitions in $\mathrm{H}_{2} \mathrm{CO}$ : (-) $2 b_{2} \rightarrow b_{2},(---) 1 b_{1} \rightarrow b_{1} ;(-\cdots---) 5 a_{1} \rightarrow a_{1} ;(\ldots \ldots-\ldots-)$ $4 a_{1} \rightarrow a_{1}$. The $1 b_{2} \rightarrow b_{2}$ cross section is negligibly small and is not included in the figure.

$\left[b_{2} \sigma(\mathrm{CH})\right.$ and $a_{1} \sigma(\mathrm{CH})$, respectively] canonical orbitals in the $\mathrm{CO}$ bond.

\section{Polarization cross sections}

The individual dipole excitation profiles of Figs. 2-6 and Tables II-IV are combined to form total verti cal electronic photoabsorption cross sections corre sponding to the three independent electric-field polarization directions in the molecular frame, associated with ${ }^{1} A_{1} \rightarrow{ }^{1} B_{1}$ ( $x$, out of plane), ${ }^{1} A_{1} \rightarrow{ }^{1} B_{2}(y$, in plane), and ${ }^{1} A_{1}-{ }^{1} A_{1}$ ( $z$, in plane) electronic transitions. Although there is no direct appropriate experimental data relat ing to these, the polarization cross sections can be compared with the previously reported CI S-T calculations. ${ }^{28}$

The discrete and continuum contributions to the appropriate cross sections are shown in Figs. 9-11, with the former given in the Stieltjes sense..$^{32-34}$ In order to construct meaningful polarization cross sections from the results of Figs. 9-11, it is necessary to perform appropriate line shape calculations for the discrete transitions lying above the first ionization threshold, since the associated states can decay through autoionization, radiationless mechanisms, and fluorescence. Although such detailed line shape calculations are not reported here, some indication of the discretestate contributions to the individual decay channels in the polarization cross sections is given in Fig. 12. The total polarization cross sections shown there are constructed employing the $\mathrm{S}-\mathrm{T}$ technique and both discrete and continuum pseudospectra, providing photoionization cross sections that correspond approximately to complete autoionization of the discrete states. By contrast, the direct photoionization cross sections shown include only the continuum contributions of Figs. $9-11$, and correspond to a situation in which the dis - 


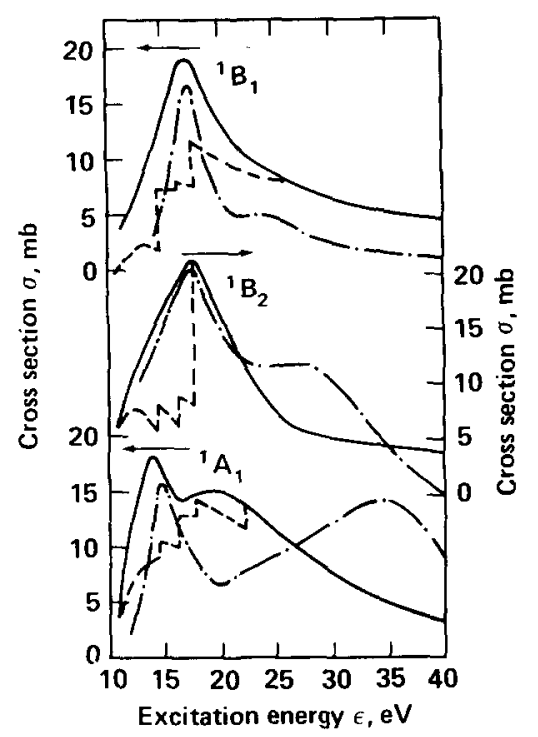

FIG. 12. Polarization cross sections for vertical electronic ${ }^{1} A_{1} \rightarrow{ }^{1} B_{1},{ }^{1} A_{1} \rightarrow{ }^{1} B_{2}$, and ${ }^{1} A_{1} \rightarrow{ }^{1} A_{1}$ excitation in molecular $\mathrm{H}_{2} \mathrm{CO}$; (-) total IVO S-T results; (-----) direct IVO S-T results; $(-\cdots,-)$ CI S-T results (Ref. 28).

crete states do not contribute to the photoionization cross sections. It is perhaps appropriate to emphasize that the cross sections of Fig. 12 do not necessarily correspond to physically observable properties, since they refer to specific polarizations in the body-fixed frame, to vertical electronic excitation that does not include the appropriate vibrational Franck-Condon or rotational London-Hönl factors, ${ }^{53}$ and embody the approximate treatment of autoionization indicated above.

\section{1. ${ }^{1} A_{1} \rightarrow{ }^{1} B_{1}$ excitations}

The individual IVO excitations contributing to the ${ }^{1} A_{1} \rightarrow{ }^{1} B_{1}$ channel shown in Fig. 9 are in generally good qualitative accord with previous excitation assignments in formaldehyde made on basis of CI S-T calculations. ${ }^{28}$ Specifically, the strong $1 b_{1} \rightarrow k a_{1}$ excitations, the $5 a_{1}$ $-k b_{1}$ excitations, and the intense $4 a_{1}-2 b_{1}$ contribution shown in the figure in the Stieltjes sense are all also evident in the CI S-T calculations. However, because of the lack of sufficient numbers of diffuse $a_{1}$ and $a_{2}$ functions in the basis set, the CI S-T calculations do not contain excitations into Rydberg $a_{1}$ or discrete and continuum $a_{2}$ orbitals. Consequently, the total IVO and CI cross sections for ${ }^{1} A_{1} \rightarrow{ }^{1} B_{1}$ excitation shown in $\mathrm{Fig}$. 12 are not in quantitative accord. However, if the $1 b_{2}$, $2 b_{2}-a_{2}$ and $1 b_{1}-n a_{1}$ contributions of Fig. 9 are subtracted from the total IVO ${ }^{1} A_{1}-{ }^{1} B_{1}$ result in Fig. 12, the two calculated cross sections are in excellent quantitative agreement. Moreover, the CI S-T results are evidently in good agreement with the direct IVO photoionization cross section in the threshold region, and the discrepancy at higher energy is accounted for by the lack of $1 b_{2}, 2 b_{2}-k a_{1}$ excitations in the CI S-T results. Evidently, the intense $4 a_{1} \rightarrow 2 b_{1}\left(\pi^{*}\right)$ transition at $\sim 17 \mathrm{eV}$ contributes substantially to the total IVO and CI photoionization cross sections for ${ }^{1} A_{1}-{ }^{1} B_{1}$ excitations. There is some indication of configuration mixing in the CI calculations in the region of the $\sim 17 \mathrm{eV}$ peak, although this has small effect on the ${ }^{1} A_{1}-{ }^{1} B_{1}$ polarization cross section.

\section{2. ${ }^{1} A_{1} \rightarrow{ }^{1} B_{2}$ excitations}

The $1 b_{2}-k a_{1}$ and $2 b_{2} \rightarrow k a_{1}$ contributions evidently dominate the IVO ${ }^{1} A_{1}-{ }^{1} B_{2}$ cross section shown in Fig. 10 , due to the presence of resonance $a_{1} \sigma^{*}(\mathrm{CO}) \mathrm{con}-$ tinuum orbitals, with smaller but nonnegligible contributions from $5 a_{1} \rightarrow k b_{2}$ and $1 b_{1} \rightarrow k a_{2}$ excitations also present. The large cross sectional value at threshold in this channel is also attributed to $2 b_{2}-k a_{1}$ excitations in the CI S-T calculations. ${ }^{28}$ Moreover, $5 a_{1} \rightarrow k b_{2}$ excitations are also weak in the CI S-T cross section, in accord with Fig. 10. There is no evidence of $1 b_{1} \rightarrow k a_{2}$ contributions to the ${ }^{1} A_{1} \rightarrow{ }^{1} B_{2}$ cross section in the CI S-T calculations, however, due to the lack of diffuse $d$ functions. Consequently, the CI S-T results shown in Fig. 12 are somewhat below the total IVO S-T calculations in the $\sim 12-20 \mathrm{eV}$ interval, although agreement is generally satisfactory. The discrete states apparently make a substantial potential contribution to the total IVO cross section for ${ }^{1} A_{1}-{ }^{1} B_{2}$ excitation in the $\sim 10-18 \mathrm{eV}$ inter val, as is evidenced by the large difference between the total and direct IVO results. Above $\sim 22 \mathrm{eV}$ excitation energy, the CI S-T calculations of Fig. 12 are somewhat larger than the IVO results for the ${ }^{1} A_{1} \rightarrow{ }^{1} B_{2}$ cross section, as a consequence of the presence of significant numbers of two-electron excitations corresponding to $\pi \rightarrow \pi^{*}$ correlation and simultaneous $1 b_{2}-k a_{1}$ or $2 b_{2}-k a_{1}$ excitation in the $\mathrm{CI}$ results. Configuration-interaction calculations in the IVO basis involving $\pi-\pi^{*}$ correlation in the ground and ionic states and the strong $1 b_{2} \rightarrow k a_{1}$ and $2 b_{2}-k a_{1}$ excitations of $\mathrm{Fig} .10$ are required to verify this effect in the ${ }^{1} A_{1} \rightarrow{ }^{1} B_{2}$ cross section. ${ }^{54}$

\section{3. ' $A_{1} \rightarrow{ }^{1} A_{1}$ excitations}

The large $2 b_{2} \rightarrow k b_{2}$ and $5 a_{1} \rightarrow k a_{1}$ IVO contributions, and the discrete $1 b_{1}-3 b_{1}$ contribution, to the ${ }^{1} A_{1}-{ }^{1} A_{1}$ polarization cross section shown in Fig. 11 are in good qualitative accord with the previous $\mathrm{CI} S-\mathrm{T}$ results. ${ }^{28}$ Moreover, there is no evidence of significant $1 b_{2}-k b_{2}$ excitations in either the CI or IVO calculations of the ${ }^{1} A_{1}-{ }^{1} A_{1}$ cross section. ${ }^{8}$ As in the CI S-T calculations, the very intense $1 b_{1}(\pi) \rightarrow 2 b_{1}\left(\pi^{*}\right)$ transition of Table $\amalg I$ is deleted in the IVO S-T results of Figs. 11 and 12, and assigned to a strong feature at $\sim 13 \mathrm{eV}$ in the total cross section (see Sec. V.E). ${ }^{50}$ The discrete $1 b_{1}-3 b_{1}$ excitation is apparently more intense and at lower excitation energy in the total IVO cross section of Fig. 12 than it is the CI cross section, accounting for the discrepancy between the two results in the $\sim 12-15 \mathrm{eV}$ interval. The $5 a_{1} \rightarrow k a_{1}$ resonance profile of $\mathrm{Fig} .11$ accounts for much of the second maximum at $\sim 18 \mathrm{eV}$ in the total IVO ${ }^{1} A_{1}$ $\rightarrow{ }^{1} A_{1}$ cross section of Fig. 12. By contrast, there is considerable configuration mixing in the CI calculations, and much of the transition intensity in the 15-25 eV excitation interval shown in Fig. 12 is evidently shifted to higher energies relative to the IVO results. This discrepancy between the CI and IVO S-T results is perhaps the most significant one in the three polarization channels, and indicates that channel coupling can be important when there are at least two strongly over- 


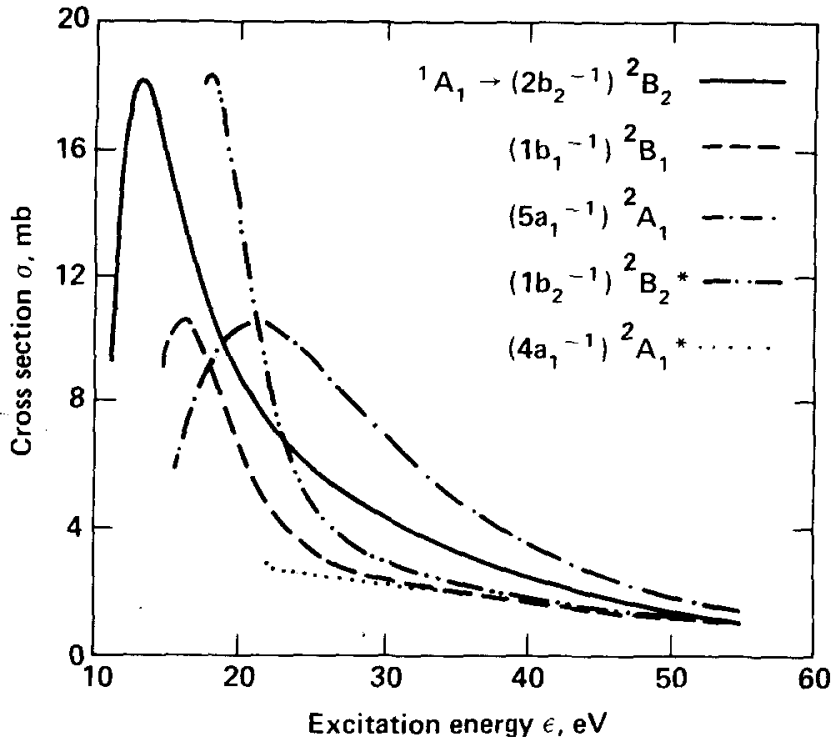

FIG. 13. Partial-channel vertical electronic photoionization cross sections in $\mathrm{H}_{2} \mathrm{CO}$ for production of the five lowest-lying electronic parent $\mathrm{H}_{2} \mathrm{CO}^{+}$molecular ionic states, constructed from the individual IVO contributions of Figs. 2-6; (-) $\left(2 b_{2}^{-1}\right)^{2} B_{2} ;(-\cdots)\left(1 b_{1}^{-1}\right)^{2} B_{1} ;(\ldots \ldots-\circ)\left(5 a_{1}^{-1}\right)^{2} A_{1} ;(\ldots \ldots \ldots)$ $\left(1 b_{2}^{-1}\right)^{2} B_{2}^{*} ;(\cdots \cdots)\left(4 a_{1}^{-1}\right)^{2} A_{1}^{*}$.

lapping intense continuum excitations present, corresponding to $2 b_{2} \rightarrow k b_{2}$ and $5 a_{1} \rightarrow k a_{1}$ transitions in the present case. Indeed, examination of the excitations contributing to the peak at $\sim 35 \mathrm{eV}$ in Fig. 12 in the CI S-T calculations reveals the presence of strong $5 a_{1} 2 b_{2}$ $\rightarrow k a_{1} k b_{2}$ contributions, as well as $5 a_{1} 1 b_{1}-2 b_{1} k a_{1}, 4 a_{1} 5 a_{1}$ $-k b_{1} k b_{1}$, and $2 b_{2} 1 b_{1} \rightarrow k b_{1} k b_{2}$ excitations. By contrast, referring to Figs. 9 and 10 , two or more intense strongly overlapping continuum excitations are apparently not present in these cases, and correspondingly the STO and $C I$ results for the ${ }^{1} A_{1}-{ }^{1} B_{1}$ and ${ }^{1} A_{1}-{ }^{1} B_{2}$ channels are in somewhat better mutual accord than are the respective ${ }^{1} A_{1}-{ }^{1} A_{1}$ cross sections.

\section{Partial-channel cross sections}

In Fig. 13 are shown the vertical electronic photoionization cross sections for the production of the five lowest-lying electronic states of the parent $\mathrm{H}_{2} \mathrm{CO}^{+} \mathrm{mo}-$ lecular ion, constructed from the individual IVO contributions of Figs. 2-6. Only the continuum contributions to the partial-channel cross sections are shown in Fig. 13 for clarity. The autoionizing states of Fig. 1 can also contribute to the partial-channel cross sections of Fig. 13, resulting in rapid variation with energy of the indicated profiles in these spectral intervals. Since the necessary autoionization line shape calculations, as well as curve-crossing phenomena, ${ }^{8}$ are not included in the present studies, the results of $\mathrm{Fig}$. 13 are not complete in the form given. Nevertheless, the very intense features in the $\left(2 b_{2}^{-1}\right)^{2} B_{2}$ and $\left(1 b_{2}^{-1}\right)^{2} B_{2}^{*}$ cross sections, and the dominance of the $\left(5 a_{1}^{-1}\right)^{2} A_{1}$ cross section at higher energy, provide unambiguous predictions for comparison with appropriate branchingratio measurements.

Although detailed partial-channel photoionization cross section measurements in $\mathrm{H}_{2} \mathrm{CO}$ over the entire excitation energy interval are apparently uriavailable at present, preliminary photoelectron spectra in the 20-30 $\mathrm{eV}$ interval have been determined. ${ }^{55}$ The branching ratios constructed from these data are compared with the present IVO predictions in Fig. 14. Although there is some scatter in the data, and it is not possible to assign experimental uncertainties in view of the subjective nature of the data analysis, ${ }^{55}$ the general agreement with the present IVO calculations is satisfactory. In particular, the ordering of the four IVO cross sections of Fig. 13 and 14 is in agreement with the measurements, and the very large $\left(5 a_{1}^{-1}\right)^{2} A_{1}$ contribution is experimentally verified in the $\sim 22-30 \mathrm{eV}$ interval. It is appropriate to note, however, that since the $5 a_{1}-k a_{1}$ contribution (Fig. 11) to the $\left(5 a_{1}^{-1}\right)^{2} A_{1}$ cross section can undergo configuration mixing with the $2 b_{2} \rightarrow k b_{2}$ channel, as previously indicated, subsequent appropriate channel-coupling calculations will perhaps modify somewhat the IVO results of Figs. 13 and 14. Moreover, since subjective procedures have been employed in their construction, ${ }^{55}$ a final date analysis may result in branching ratios that differ somewhat from the experimental results of Fig. 14 .

\section{E. Total photoabsorption/ionization cross sections}

The polarization cross sections of Fig. 12 are combined and compared in Fig. 15 with the previously reported CI S-T results ${ }^{28}$ and with the available measured cross sectional data., 8 The photoionization mass-spectrometric measurements ${ }^{8}$ are placed on an absolute scale by normalizing to the measured photoabsorption cross section at $\sim 12.3 \mathrm{eV},{ }^{4}$ at which energy photoionization apparently dominates the total photoabsorption cross section. When this procedure is employed, the resulting photoionization cross section for $\mathrm{H}_{2} \mathrm{CO}^{+}$production is apparently larger than the total measured

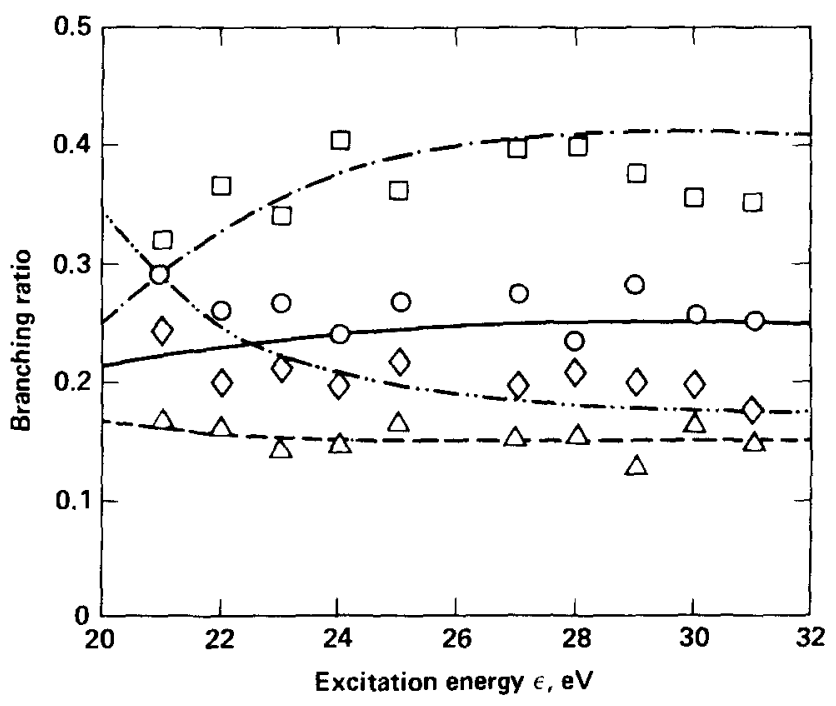

FIG. 14. Photoionization branching ratios in $\mathrm{H}_{2} \mathrm{CO}$ for production of the four lowest-lying electronic parent $\mathrm{H}_{2} \mathrm{CO}^{+}$molecular ionic states; $\left(2 b_{2}^{-1}\right)^{2} B_{2},(-)$ IVO S-T, (๑) experiment; $\left(1 b_{1}^{-1}\right)^{2} B_{1}$, (-.--) IVO S-T, (४) experiment; $\left(5 a_{1}^{-1}\right)^{2} A_{1},(-\cdot-\infty)$ IVO S-T, (a) experiment; $\left(1 b_{2}^{-1}\right)^{2} B_{2}^{*},(-\cdots-\cdots-)$ IVO $S-T$, (•) experiment. Experimental values constructed from photoelectron spectra kindly provided by $T$. Gustafsson (Ref. 55 ). 


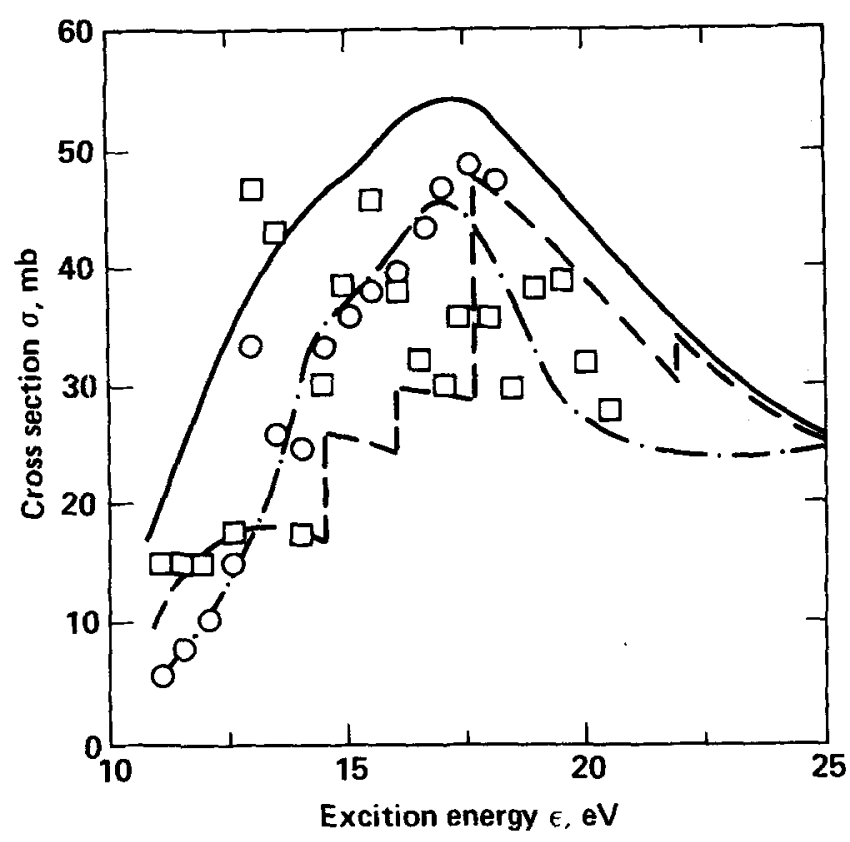

FIG. 15. Photoabsorption/ionization cross sections in $\mathrm{H}_{2} \mathrm{CO}$ : (-) total vertical electronic IVO S-T cross section constructed from the total polarization cross sections of Fig. 12; (-----) di rect vertical electronic IVO S-T photoionization cross section; (-.....-) vertical electronic CI S-T cross section (Ref. $28)$; (•) photoionization-mass-spectrometric measurement (Ref. 8) normalized to the total photoabsorption cross section (Ref. 4) at $\sim 12.3 \mathrm{eV}$; ( $\backsim$ ) measured total photoabsorption cross section (Ref. 4). Experimental uncertainties of $\sim \pm 30 \%$ are suggested.

photoabsorption cross section at higher energies. This experimental discrepancy prevents a complete and mean ingful detailed comparison with the present calculations. ${ }^{56}$ Nevertheless, since the IVO and CI results are compatible, and there is some degree of agreement between the experimental and theoretical results, general comparative remarks are in order.

The discrepancy between the IVO and CI S-T total cross sections of Fig, 15 can be attributed to basis-set differences, and possibly some degree of configuration mixing in the $\sim 15-25 \mathrm{eV}$ interval, as indicated in a previous subsection (Sec. V.C). In view of the complexities of the two independent calculations, and the rich underlying structure (Figs. 2-6) of the total cross section, the agreement between the two total theoretical results shown in Fig. 15 is quite satisfactory. The total photoabsorption cross section measurements, how ever, which presumably include all neutral and ionic final channels, are generally below the total IVO S-T result, and are also below the direct IVO and CI S-T results at certain energies. Since the total measured photoionization cross section (not shown) falls below the total photoabsorption cross section, it is by necessity also generally below the calculated values. Nevertheless, the measured cross sections are generally within a factor of 2 of the calculations, and are in better accord at certain energies. The structure at $-13 \mathrm{eV}$ is a suitable candidate for the missing $\pi \rightarrow \pi^{*}$ resonance transition, which is possibly placed at this relatively high energy in the spectrum by a broad bound-to-continuum Franck-Condon factor and vibronic predissociation and preionization. ${ }^{8,28,50}$

In order that the vertical electronic direct IVO S-T results of $F$ ig. 15 correspond to the measured values for $\mathrm{H}_{2} \mathrm{CO}^{+}$production, it is necessary that the FranckCondon factors for the individual ionic states treated do not extend significantly above the dissociation thresholds, and that the contributions from autoionization be gene rally small. The measured high resolution (584 $\AA$ ) photoelectron spectra in $\mathrm{H}_{2} \mathrm{CO}$ suggest there are only small contributions to dissociative photoionization from the lowest four one-electron ionization processes. ${ }^{2}$ Evidently, the direct vertical electronic IVO results of Fig. 15 are in general accord with the measured cross section for $\mathrm{H}_{2} \mathrm{CO}^{*}$ production at threshold and at the $\sim 17.5 \mathrm{eV}$ peak, although there is some evidence of contributions from autoionization in the $\sim 14-17 \mathrm{eV}$ interval. The CI S-T cross section is also in accord with the $\mathrm{H}_{2} \mathrm{CO}^{+}$cross section, although the very good agree ment indicated must be regarded as somewhat fortuitous in view of the basis set deficiencies present and in light of the discrepancies between the experimental measurements. ${ }^{4,6}$ Further measurements and refined calculations including effects of the vibrational degrees of freedom, autoionization, and the contribution of the $\pi \rightarrow \pi^{*}$ transition are required for a definitive comparison between theory and experiment.

\section{CONCLUDING REMARKS}

The theoretical studies of vertical electronic photoexcitation and ionization in molecular formaldehyde reported here employ square-integrable functions only and the recently devised Stieltjes-Tchebycheff technique. The cross sections obtained correspond to results from conventional bound - and continuum-state calculations in the improved-virtual-orbital or static-exchange approximation. The importance of correct treatments of continuum molecular orbitals of good symmetry type in formaldehyde is emphasized by the presence of finalstate resonance orbitals in many of the photoionization channels. These generally cannot be predicted by use of plane-wave and Coulombic final states, and partialwave expansions are expected to be slowly convergent in this case. Although precise calculations involving the full nonlocal, noncentral molecular Fock potential are apparently required for quantitatively reliable predictions of molecular photoexcitation and ionization cross sections, the appearance of resonance features in the photoionization continua can be anticipated qualitatively on basis of the numbers and types of valencelike virtual orbitals available for occupancy. In particular, $k a_{1} \sigma^{*}(\mathrm{CO})$ photoionization resonances are apparently prevalent in molecular formaldehyde, and in the continuous spectrum of the previously studied $\mathrm{CO}$ chromophore, and there is good evidence also for the presence of $k b_{2} \pi_{y}^{*}(\mathrm{CO})$ resonances in formaldehyde. The prediction of these features and their qualitative description in terms of conventional valencelike $\sigma^{*}$ and $\pi_{y}^{*}$ vir tual orbitals in the photoionization continuum is perhaps one of the more noteworthy aspects of the present study. 
The calculated $2 b_{2}(n)$ improved -virtual -orbital spectra are in generally good agreement with available position and intensity measurements, although certain ( $n d$, $n f)$ series are not included in the present calculations. Moreover, although the predicted positions of the higher spectral series involving excitations of $1 b_{1}(\pi), 5 a_{1}(\sigma)$, $1 b_{2}$, and $4 a_{1}$ orbitals are in general agreement with assignments made primarily on basis of measured ionization potentials and expected quantum defects, it is clear that appropriate line shape calculations are required to properly account for intensity distributions in autoionization regions. In particular, the $\pi \rightarrow \pi^{*}$ transition is expected to significantly perturb intensity distributions in the ${ }^{1} A_{1} \rightarrow{ }^{1} A_{1}$ electronic channel, and perhaps in other electronic channels as well, as a consequence of the nonplanar and dissociative nature of the excited state in this case. Such intensity perturbations presumably account for the apparent absence in the experimental spectra of the moderately strong $n=3$ and other members of Rydberg series involving $1 b_{1}(\pi)$ and $5 a_{1}(\sigma)$ excitations. Consequently, the present calculations are not complete in the regions of autoionization above the first ionization potential, but can provide useful zeroth-order approximations in these regions for subsequent appropriate line shape studies.

The individual -channel photoionization cross sections obtained in the static-exchange (IVO) approximation should provide reliable one-electron vertical excitation profiles in those cases in which channel coupling is small. With the possible exception of interactions between strong $2 b_{2} \rightarrow k b_{2}$ and $5 a_{1}-k a_{1}$ excitations in the ${ }^{1} A_{1} \rightarrow{ }^{1} A_{1}$ electronic channel, coupling among the various one-electron photoionization continua is expected to be generally weak. By contrast, as indicated above, autoionizing decay of the various higher discrete states can produce rapid variation with energy in the partial channel cross sections. The predicted orderings in intensities of the partial-channel photoionization cross sections for production of the four lowest-lying parent ion states are in good agreement with recent synchrotron-radiation photoelectron branching-ratio measure ments in the $20-30 \mathrm{eV}$ interval. Most important in this connection is the tentative verification of a very strong broad feature in the $\left(5 a_{1}^{-1}\right)^{2} A_{1}$ channel attributed to the presence of $k a_{1} \sigma^{*}(\mathrm{CO})$ valencelike resonance orbitals in the continuum.

When the various individual-channel cross sections a re appropriately combined, polarization cross sections in the body frame for ${ }^{1} A_{1} \rightarrow{ }^{1} B_{1},{ }^{1} B_{2}$, and ${ }^{1} A_{1}$ electronic excitations are obtained that are in generally good agreement with the corresponding results of previously reported CI (S-T) calculations. Although there are some quantitative discrepancies in the respective results for the ${ }^{1} A_{1}-{ }^{1} A_{1}$ cross section, due apparently to channel coupling, the overall agreement is quite satisfactory in view of the general differences in the two independent calculations and in light of the highly structured natures of the individual IVO spectra. Particularly good qualitative agreement between the two calculations is obtained for electron configurational assignments in the continuum.
The calculated total vertical electronic photoabsorption and ionization cross sections are significantly less structured than are the individual excitation channels, and they do not reveal the presence of the various resonance features present in the photoionization continua. In spite of quantitative discrepancies between the total photoabsorption and mass-spectrometric photoionization measurements, and lack of detailed theoretical treatment of autoionization, fluorescence, and radiationless decay phenomena, the theoretical and experimental results are in general qualitative accord. It would seem therefore that a reliable approximate account of vertical electronic dipole excitation and ionization intensities and assignments in molecular formaldehyde has been achieved on basis of the present IVO $(\mathrm{S}-\mathrm{T})$ calculations.

\section{ACKNOWLEDGMENTS}

This work was performed under the auspices of the U. S. Department of Energy under Contract No. W-7405-Eng-48. One of us (B. V. M.) acknowledges the support provided by a grant from the National Science Foundation and another (P. W. L.) acknowledges the support provided by a grant from the Donors of the Petroleum Research Fund, administered by the American Chemical Society.

${ }^{1}$ G. H. Dieke and G. B. Kistiakowsky, Phys. Rev. 45, 4 (1934).

${ }^{2}$ A. D. Baker, C. Baker, C. R. Brundle, and D. W. Turner, Int. J. Mass Spectrom. Ion Phys. 1, 285 (1968).

${ }^{3} \mathrm{M}$. Praet and J. Delwiche, Int. J. Mass Spectrom. Ion Phys. 1, 321 (1968).

${ }^{4} J$. E. Mentall, E. P. Gentieu, M. Krauss, and D. Newmann, J. Chem. Phys. 55, 5471 (1971).

${ }^{5}$ M. J. Weiss, C. F. Kuyatt, and S. Mielczarek, J. Chem. Phys. 54, 4147 (1971).

${ }^{6}$ C. R. Lessard, D. C. Moule, and S. Bell, Chem. Phys. Lett. 29, 603 (1974).

${ }^{7}$ D. C. Moule and A. D. Walsh, Chem. Rev. 75, 67 (1975).

${ }^{8}$ P. -M. Guyon, W. A. Chupka, and J. Berkowitz, J. Chem. Phys. 64, 1419 (1976).

${ }^{9}$ S. T. Hood, A. Hamnett, and C. E. Brion, Chem. Phys. Lett. 41, $428(1976)$.

${ }^{10}$ C. R. Drury-Lessard and D. C. Moule, Chem. Phys. Lett. 47, 300 (1977); J. Chem. Phys. 66, 3908 (1977).

${ }^{11} \mathrm{M}$. B. Robin, Higher Excited States of Polyatomic Molecules (Academic, New York, 1974), Vol. II.

${ }^{12}$ T. H. Dunning, Jr. and V. MeKoy, J. Chem. Phys. 47, 1735 (1967).

${ }^{13}$ J. L. Whitten and M. Hackmeyer, J. Chem. Phys. 51, 5584 (1969).

${ }^{14}$ R. J. Buenker and S. D. Peyerimhoff, J. Chem. Phys. 53, $1368(1970)$

${ }^{15}$ S. D. Peyerimhoff, R. J. Buenker, W. E. Krammer, and H. Hsu, Chem. Phys. Lett. 8, 129 (1971).

${ }^{16}$ J. L. Whitten, J. Chem. Phys. 66, 5458 (1972).

${ }^{17}$ I. Absar, C. S. Lin, and K. L. McEwen, Can. J. Chem. 50, 646 (1971).

${ }^{18}$ J. C. Ho, G. A. Segal, and H. S. Taylor, J. Chem. Phys. 56, $1520(1972)$.

${ }^{19}$ B. J. Garrison, H. F. Schaefer, and W. A. Lester, J. Chem. Phys. 61, 3039 (1974).

${ }^{20} \mathrm{D}$. L. Yeager and V. McKoy, J. Chem. Phys. 60, 2714 (1974).

${ }^{21}$ S. R. Langhoff, S. T. Elbert, C. F. Jackels, and E. R.

Davidson, Chem. Phys. Lett. 29, 247 (1974).

${ }^{22}$ T. D. Davis, G. M. Maggiora, and R. E. Christoffersen, J. Am. Chem. Soc. 96, 7878 (1974).

${ }^{23}$ S. D. Peyerimhoff and R. J. Buenker, Advan. Quantum Chem. 9, 69 (1975). 
${ }^{24}$ K. J. Miller, J. Chem. Phys. 62, 1759 (1975).

${ }^{25} \mathrm{~S}$. R. Langhoff and C. W. Kern, in Modern Theoretical Chemistry IV. Applications of Electronic Structure Theory, edited by H. F. Schaefer (Plenum, New York, 1975), Chap. XII.

${ }^{26}$ L. B. Harding and W. A. Goddard, J. Am. Chem. Soc. 97, 6293 (1975); 99, 677 (1977).

${ }^{27}$ S. R. Langhoff and E. R. Davidson, J. Chem. Phys. 64, 4699 (1976).

${ }^{28} \mathrm{P}$. W. Langhoff, S. R. Langhoff, and C. T. Corcoran, J. Chem. Phys. 67, 1722 (1977).

${ }^{29}$ T. N. Rescigno and P. W. Langhoff, Chem. Phys. Lett. 51, 65 (1977).

${ }^{30}$ T. N. Rescigno, C. F. Bender, B. V. McKoy, and P. W. Langhoff, J. Chem. Phys. 68, 970 (1978).

${ }^{31}$ N. Padial, G. Csanak, B. V. McKoy, and P. W. Langhoff, J. Chem. Phys. 69, 2992 (1978).

${ }^{32}$ P. W. Langhoff, Chem. Phys. Lett. 22, 60 (1973).

${ }^{33}$ P. W. Langhoff and C. T. Corcoran, J. Chem. Phys. 61, 146 (1974).

${ }^{34} \mathrm{P}$. W. Langhoff, J. Sims, and C. T. Corcoran, Phys. Rev. A 10, 829 (1974).

${ }^{35}$ P. W. Langhoff and C. T. Corcoran, Chem. Phys. Lett. 40, 367 (1976)

${ }^{36}$ P. W. Langhoff, C. T. Corcoran, J. S. Sims, F. Weinhold, and R. M. Glover, Phys. Rev. A 14, 1042 (1976).

${ }^{37}$ C. T. Corcoran and P. W. Langhoff, J. Math. Phys. 18, 651 (1977).

${ }^{38}$ P. W. Langhoff, C. T. Corcoran, and J. S. Sims, Phys. Rev. A 16,1513 (1977).

${ }^{39}$ P. W. Langhoff, Int. J. Quantum Chem. Symp. 11, 301 (1977).

${ }^{40} \mathrm{P}$. W. Langhoff, J. Chem. Phys. 57, 2604 (1972).

${ }^{41} \mathrm{C}$. T. Corcoran and P. W. Langhoff, Chem. Phys. Lett. 41, 609 (1975).

${ }^{42}$ P. M. Johnson, P. W. Langhoff, S. V. ONeil, C. T. Corcoran, and W. P. Reinhardt, Chem. Phys. Lett. 52, $380(1977)$.

${ }^{43}$ W. J. Hunt and W. A. Goddard, Chem. Phys. Lett. 3, 414 (1969); 24, 464 (1974).

${ }^{44}$ H. P. Kelly, Phys. Rev. B 136, 896 (1964).

${ }^{45}$ P. W. Langhoff, M. Karplus, and R. P. Hurst, J. Chem. Phys. 44, 505 (1966).

${ }^{46}$ P. W. Langhoff and S. W. Chan, Mol. Phys. 25, 345 (1973).

${ }^{47}$ T. H. Dunning, Jr. and N. W. Winter, J. Chem. Phys. 55, 3360 (1971).

${ }^{48}$ It is perhaps appropriate to note that the Rydberg or valence nature of a transition cannot, of course, be decided on the basis of effective principal quantum number alone. Nevertheless, $n^{*}$ values provide readily available suggestive criteria for identifying Rydberg transitions when employed in conjunction with the generally accepted quantum defects of $\sim 1.0$, 0.7 , and 0.1 for $s, p$, and $d$ series, respectively. See, for example, the remarks in this connection of M. B. Robin, "Aspects of Molecular Rydberg States," in Chemical Spectroscopy and Photochemistry in the Vacuum Ultraviolet, edited by C. Sandorfy, P. J. Ausloos, and M. B. Robin (Reidel, Boston, 1974), pp. 13-23; and in Vol. I of Ref. 11.

${ }^{49}$ The $3 s, 3 p, 3 d$, and $4 f$ orbitals of light atoms can mix strongly with valencelike $(2 s, 2 p)$ molecular orbitals, and are conveniently designated as "pre-Rydberg" in character. See in this connection the discussion of $O$. Sinanoglu, "Theory of Intravalency and Rydberg Transitions in Molecules," in Chemical Spectroscopy and Photochemistry in the Vacuum Ultraviolet, edited by C. Sandorfy, P. J. Ausloss, and M. B. Robin (Reidel, Boston, 1974), pp. 337-384.

${ }^{50}$ The missing $\pi \rightarrow \pi^{*}$ transition in formaldehyde remains a somewhat troublesome feature of its spectrum, although an assignment in general accord with the theoretical predictions. [L. Z. Stenkamp and E. R. Davidson, Theor. Chim. Acta 44, 405 (1977); and L. E. Nitzsche and E. R. Davidson, J. Chem. Phys. 68, 3103 (1978)] has been made of the corresponding transition in formamide (Ref. 11), and the recent assignment of the $\pi \rightarrow \pi^{*}$ transition in thioformaldehyde [R. H.
Judge, C. R. Drury-Lessard, and D. C. Moule, Chem. Phys. Lett. 53, $82(1978)$ is in good accord with the calculations of S. D. Peyerimhoff and R. J. Buenker, "Ab Initio Calculations for Excited States of Molecules," in Chemical Spectroscopy and Photochemistry in the Vacuum Ultraviolet, edited by C. Sandorfy, P. J. Ausloss, and M. B. Robin (Reidel, Boston, 1974), pp. 257-286. In the latter case, the observed transition is quite broad, and underlies sharper Rydberg transitions. Since the corresponding upper state in $\mathrm{H}_{2} \mathrm{CO}$ is presumably nonplanar (Ref. 7) and dissociative in the C-O stretch (Ref. 14), reflection of the ground state vibrational function in the upper-state potential, vibronic preionization, and competing dissociation can account for a substantial broadening and shift from the theoretically predicted position in this case. Although these observations are in accord with the photoionization-mass-spectrometric studies of P. -M. Guyon et al . (Ref. 8), and with the ionization efficiency measurements of M. Praet and J. Delwiche (Ref. 3), detailed vibronic coupling and autoionization calculations are required to clarify the nature of the $\pi \rightarrow \pi^{*}$ transition in $\mathrm{H}_{2} \mathrm{CO}$.

${ }^{51}$ Weak resonances in the $2 b_{2} \rightarrow k a_{2}, 1 b_{1} \rightarrow k a_{2}$, and $1 b_{2} \rightarrow k a_{2}$ channels apparently involve somewhat subtle interactions between $3 d$ pre-Rydberg and valence-basis orbitals, as indicated above (Ref. 49). Consequently, although the present study provides a quantitative description of these features, further qualitative clarification will require detailed examination of the spatial characteristics of the appropriate orbitals, rather than a simpler discussion involving valence-basis molecular orbitals alone.

${ }^{52}$ It is perhaps appropriate to note that qualitative clarification of the origins of resonances in molecular photoionization is also obtained from conventional one-center scattering considerations involving penetration of an appropriate centrifugal barrier by higher angular-momentum waves. See, for example, the discussions of J. L. Dehmer and D. Dill, J. Chem. Phys. 65, 5327 (1976); and J. W. Davenport, Int. J. Quantum Chem. Symp. 11, 89 (1977), in the respective cases of $K-$ shell and valence photoionization resonances in diatomic molecules. Such descriptions are perhaps somewhat less predictive in cases of polyatomic molecules than is the qualitative argument given here involving valence-basis determinations of virtual molecular orbitals of good symmetry type.

${ }^{53} \mathrm{G}$. Herzberg, Molecular Spectra and Molecular Structure (Van Nostrand, New York, 1966), Part III.

${ }^{54} \mathrm{As}$ indicated in Ref. 28, the $1 b_{1} 2 b_{2} \rightarrow k a_{1} 2 b_{1}$ excitations in the CI S-T ${ }^{1} B_{2}$ spectrum can also be regarded as $n-\pi^{*}$ correlation with simultaneous $1 b_{1} \rightarrow k a_{2}$ excitation. The strong $1 b_{2}$ $\rightarrow k a_{1}$ and $2 b_{2} \rightarrow k a_{1}$ dipole allowed IVO spectra of Fig. 10, however, suggest this view is perhaps less appropriate than is the present view involving $\pi \rightarrow \pi^{*}$ correlation in the ground and ionic states.

${ }^{55}$ We are grateful to T. Gustafsson and E. W. Plummer for providing us with measured photoelectron spectra in formaldehyde, from which we have constructed the preliminary branching ratios of $\mathrm{Fig}$. 14. We emphasize in this connection that the experimental results of Fig. 14 are of a very preliminary nature, and final values will require a more detailed data analysis. Specifically, an appropriate instrument function was not available to us, and so a highly subjective procedure was employed in subtracting background contributions from the measured photoelectron spectra. Moreover, no attempt has been made to correct the experimental results of Fig. 14 for anisotropy effects, a highly subjective separation of the overlapping $5 a_{1}$ and $1 b_{2}$ photoelectron bands has been made, and the possible effects of higher-lying electronic states have not been included in the analysis. In spite of the preliminary nature of these branching ratios, it was felt appropriate to present them here for comparison with the IVO calculations.

${ }^{56}$ Electron-impact-excitation studies presently underway should aid in clarifying the discrepancy [R. A. Bonham (private communication)]. 